\title{
Spontaneous bulk organization of molecular assemblers based on aliphatic polyether and/or poly(benzyl ether) dendrons
}

\author{
Byoung-Ki Cho
}

This paper reviews our recent research with regard to bulk self-assemblers based on well-defined branched building blocks. Branched molecules/polymers have been known to show different physical properties from conventional linear-shaped polymers. As well as thermal and solution properties, structural modification into branched building blocks also has a significant influence on self-assembling behavior in the bulk states, including solid and liquid crystalline (LC) phases. As a branched component, dendrons/dendrimers with well-defined structures can be ideal candidates for the construction of advanced self-assemblers. Recently, we worked on new assembling systems such as dendron-coils, block codendrimers and discotic LC molecules. In the design, we used aliphatic polyether dendrons in most cases and poly(benzyl ether) dendrons in part. Their bulk assembling and thermal properties were found to be distinct from conventional linear block copolymers and they were dependent upon their chain architectures. To self-assemble into ordered nanostructures, the hybrid assembers were designed to have incompatible blocks, for example, hydrophilic and hydrophobic parts. Furthermore, in some cases an ionic complexation was carried out to maximize the immiscibility between the two different blocks. This review describes the molecular manipulations to engineer the nanoassemblies in bulk and also the ionic transportation properties depending on the assembling morphology.

Polymer Journal (2012) 44, 475-489; doi:10.1038/pj.2012.31; published online 28 March 2012

Keywords: bulk assembly; codendrimers; dendron; discotic liquid crystals; self-assembler

\section{INTRODUCTION}

Much attention has been given to the generation of ordered morphologies by self-assemblers because of the tremendous potential for the creation of functional materials. ${ }^{1-3}$ The morphological property of each nanostructure is dependent upon the identity of organizing building blocks and can be manipulated by elaborate engineering of related molecular parameters, such as polarity, stiffness and chain architecture. ${ }^{4}$ Therefore, a hybridization approach of architecturally different components with their own organizing features can be a powerful tool to explore a new class of self-assemblers.

Variation of the macromolecular chain architecture into unconventional types influences physical properties, such as viscosity, hydrodynamic volume, thermal and assembling behavior. ${ }^{5}$ In particular, branched polymers have been studied as a counterpart to linear polymers to identify distinct physical behavior. ${ }^{6}$ As representative branched examples, dendrimers and dendrons (sections of dendrimers) have well-defined tree-like chain structures with lowmolecular-weight distributions. Because of their refined structural features, they have been used as useful macromolecular building blocks for functional materials. ${ }^{7}$

Having two chemically distinct compartments, the peripheries and the dendritic core, can offer ordered assemblies in the bulk state, and in most cases, micellar and cylindrical morphologies were found due to their typical cone or tapered molecular shape. The Percec-type poly(benzyl ether) dendrimers/dendrons have been extensively studied as a representative dendritic self-assembler in the liquid crystalline (LC) state. ${ }^{8}$ Because of the conformational difference between rigid benzyl units and flexible alkyl peripheries, ordered LC morphologies can be formed. In addition, by modification of the dendron generation, focal groups and number/site of peripheral groups on aromatic units, the shape of poly(benzyl ether) dendritic molecules can be engineered to manipulate the LC structures.

Besides the aromatic dendritic system, an aliphatic polyether dendritic system has recently been used as a self-assembling building unit. Despite its weak assembling power, rational molecular modifications can lead to ordered structures in the bulk state. Recently, we reported several assembling examples based on aliphatic polyether dendrons. $^{9-11}$ To highlight this relatively unexplored dendritic unit, we may mention several remarkable properties of this aliphatic system distinguishable from aromatic dendrons. First of all, as compared with conventional poly(benzyl ether) dendrons, aliphatic polyether dendrons free of any labile linkage are chemically and thermally stable. Thus, this dendritic system might be suitable for the combination with other building blocks, and can be an alternative 
dendritic building block for new self-assemblers with unconventional architectures. For instance, an assembling block copolymer based on aliphatic polyether dendritic system can be manipulated under harsh conditions such as high temperatures. This may provide opportunities to study assembling and functional properties, which cannot be accessed in labile assembling systems. As well as the structural stability, the flexible conformational feature of aliphatic polyether dendritic blocks is advantageous to obtain rich assembling properties in conjugated assembling systems, although no LC phase can be expected on their own. Flexible dendritic blocks can readily transform their conformations into various shapes in response to certain stimuli (for example, temperature), leading to diverse assembling morphologies. Therefore, in comparison with shapepersisted aromatic dendrons, aliphatic polyether dendron-based assemblers may expect richer assembling and materials functions, which is useful for development of functional dendritic materials.

For the last few years, our research has been focused on the creation of a novel class of self-assembling materials based on the aliphatic polyether and/or the Percec-type dendritic systems. By combining the dendrons with architecturally different blocks, some hybrid selfassemblers could be prepared and their assembling properties were investigated. For example, copolymers with a dendron-coil architecture were examined extensively in the solid and LC states. We could engineer the local properties of the dendritic block (for example, the introduction of hydrophilic peripheries instead of hydrophobic ones) by which the physical nature of the dendritic unit could be modulated. In addition, by linking two physically different dendron blocks, a Janus-type dendrimer system (referred to as block codendrimers) could be obtained, and the LC behavior of these dendrimers could be examined upon ion doping.

Although several dendron-based hybrid systems have been reported to date, most of them did not show a well-ordered LC phase, presumably because of the weak segregation between the blocks. ${ }^{12-14}$ To overcome the weakness of previous examples, we focused on the design of highly amphiphilic molecular systems, which form ordered morphologies in the LC states. As a way to fine-tune the morphological and functional features in the LC phases, we chose and coupled appropriate molecular candidates when preparing hybrid assemblers. For a particular purpose, this approach could be used to design suitable functional assemblers. Briefly speaking, we can use a molecular tool box consisting of diverse building blocks with different shapes and physical properties to devise a target self-assembler for specific assembling and/or functional properties. For example, an aliphatic polyether dendron was chosen for ion-conducting dendroncoils. Because of its chemical stability and hydrophilicity, the aliphatic component can be proper candidate for electrolytes. By considering enhanced ion conduction, dendron blocks have a strong tendency to locate a continuous matrix region in dendron-coil assemblies, leading to three-dimensional movement of ions. In this way, our rational molecular designs were performed to vary diverse molecular parameters for the systematic control of LC morphologies and the related materials function such as ionic transportation in the LC phase.

As appears in the title, the dendritic blocks are the core component for designing molecular assemblers in our research, and we investigated their assembling and functional roles in hybrid molecular systems. So far, we have mainly investigated the bulk properties of molecular assemblers, which are addressed in this review paper. Nevertheless, some molecular assemblers with strong amphiphilicity possibly organize into various ordered morphologies in solution phase. Therefore, we are convinced that our assemblers can broaden research boundaries to novel solution applications such as drug delivery and supramolecular sensors, although not described in this paper.

\section{ALIPHATIC POLYETHER DENDRONS FUNCTIONALIZED WITH ALKYL PERIPHERIES}

In comparison to the Percec-type poly(benzyl ether) dendrons, aliphatic polyether dendrons have less organizing power, because of the higher flexibility in their linkages. To generate ordered organization in bulk, special molecular operations are required. To do this, the peripheral site could be functionalized with chemically distinct hydrophobic alkyl chains from the polar dendritic polyether core. In 2004, Cho and Wiesner synthesized a series of aliphatic polyether dendrons with hydrophobic docosyl peripheries and investigated the solid state assemblies of the dendrons as a function of dendron generation (Figure 1). ${ }^{9}$ The low-generation dendrons (1-3) showed a bilayered lamellar morphology with an interdigitated core packing, whereas the highest generation (4) exhibited an oblique columnar assembly (Figure 2). This assembling behavior can be explained by the fact that the molecular shape becomes isotropic with increasing dendron generation.

In addition to the variation of dendron generation, another method to control the dendron shape is the manipulation of the peripheral alkyl chain length. In the crystalline state, the reduction of the alkyl chain length is expected to make the dendron shapeless anisotropic. ${ }^{11}$ Therefore, at a given generation, the crystalline morphology can be tuned depending on the alkyl chain length. ${ }^{10}$ We fixed the dendron generation to the third generation, but the alkyl chain changed from docosyl (3), to octadecyl (5) to tetradecyl (6) group (Figure 1). Despite being the same generation, the dendrons with the shorter octadecyl and tetradecyl chains displayed the oblique columnar structure, which is in contrast to the lamellar structure from the dendron with docosyl peripheries (Figure 2).

Consequently, the solid state assembling behavior of the aliphatic polyether dendron system can be engineered by either dendron generation or peripheral alkyl chain length.

\section{AMPHIPHILIC DENDRON-COILS BASED UPON ALIPHATIC POLYETHER DENDRONS}

The hybridization of two molecular extremes, that is, branched dendron and linear coil, might lead to an interesting assembling system because these blocks are known to have distinct selfassembling behavior. In the early stage, the aim was focused on the construction of the dendron-coil molecular architecture by using diverse synthetic strategies ${ }^{15-17}$ or the study of the self-assemblies in solution. ${ }^{18-20}$ However, the recent interest has shifted to the bulk assemblies of dendron-coil molecules. Many dendron-coil assembling systems were reported, but they mostly addressed the solid state.

A careful selection of block compositions, such as a hydrophobic alkyl chain and a hydrophilic coil, can lead to a novel self-assembling system in the LC state. ${ }^{21}$ In this line, the above-mentioned aliphatic polyether dendritic system could be extended by attaching a linear hydrophilic coil. Cho and Wiesner designed a dendron-coil system composed of a third-generation aliphatic polyether dendron with docosyl peripheries and a linear poly(ethylene oxide) (PEO) coil (Figure 3a). ${ }^{22}$ By binding the hydrophilic PEO coil at the focal position of the dendron, the amphiphilic character could be maximized, by which molten LC phases were obtained. The morphologies in the LC state were systematically controlled by changing the PEO coil length. The dendron coil (7) with the shorter PEO coil (degree of polymerization of $\mathrm{PEO}=31$ ) showed a 

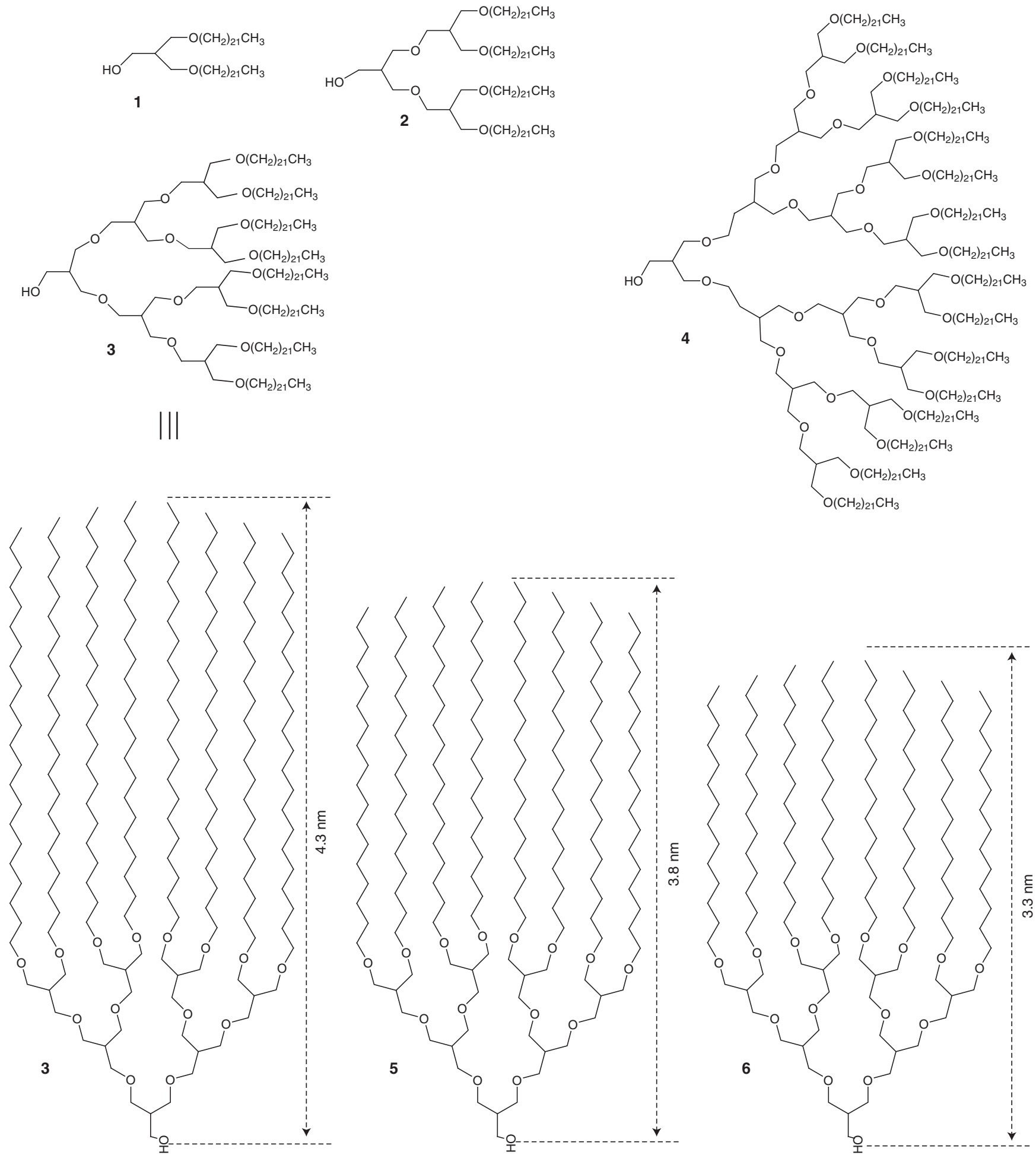

Figure 1 Molecular structures of aliphatic polyether dendrons 1-6.

micellar phase with Pm3n symmetry, which is referred to as the A15 micellar phase (Figure 3b). ${ }^{23}$ This LC micellar morphology has a different micellar packing characteristic from the body-centered cubic with $\operatorname{Im} 3 \mathrm{~m}$ symmetry observed in conventional linear block copolymers, and has been observed in only a few poly(benzyl ether) dendrons. ${ }^{24,25}$ On the other hand, the dendron-coil (8) with a longer
PEO coil (degree of polymerization of $\mathrm{PEO}=96$ ) exhibited three LC phases-columnar, continuous cubic (gyroid) and lamellar structures-as a function of temperature (Figure 3c). Particularly, among the observed morphologies, the gyroid LC phase has been frequently observed in linear block copolymers, ${ }^{26}$ but has not been seen in dendrons/dendrimers. Considering the A15 and gyroid 
morphologies, we can conclude that this dendron-coil system is a unique assembling system that shares the morphological features of linear block copolymer and dendrimer/dendron systems.

Recently, our group investigated a modified dendron-coil system in which the dendron generation of the aliphatic polyether dendron with octadecyl peripheries varied from first, to second, to third at a fixed PEO coil with a molecular weight of $2000 \mathrm{~g} \mathrm{~mol}^{-1}$ (Figure 4). ${ }^{27}$ From this dendron-coil system, we demonstrated the generation dependence of the assembling behavior in both crystalline and LC states. The dendron-coils exhibited two crystalline states because both alkyl and PEO blocks are crystalline (Figure 5). Although all the crystalline states were shown to have a lamellar morphology, the crystalline lamellar spacing upon PEO melting changed differently depending on the dendron generation. The lamellar spacing of

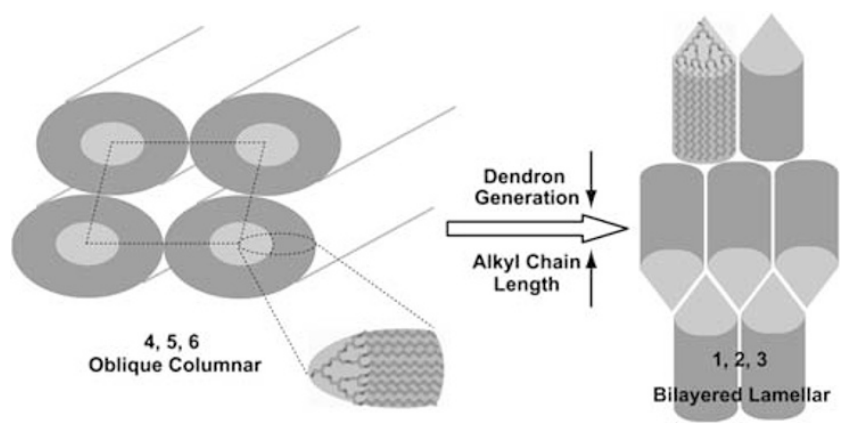

Figure 2 Solid state assemblies of aliphatic polyether dendrons depending on the dendron generation or alkyl chain length. dendron-coil 9 decreased upon the PEO melting, whereas dendroncoil 11 exhibited a larger lamellar spacing (Figure 5). And, the lamellar spacing of dendron-coil $\mathbf{1 0}$ with the intermediate second generation did not change at the PEO melting transition (Figure 5). The generation dependency of the lamellar spacing upon PEO melting is attributed to the interplay between the conformation stabilization and the specific volume increase in the molten PEO coil. The former conformation issue dominant in dendron-coil 9 expands the coil cross-section laterally in order to reduce the coil stretching penalty. However, the increased PEO-specific volume upon melting results in the vertical expansion of the lamellar spacing of dendroncoil 11. This is because no lateral expansion is necessary in the third generation with a larger cross-section. For dendron-coil 10, these two competitive factors might be neutralized, thus no change in the spacing occurred.

Upon melting of the alkyl peripheries, two dendron-coils (10 and 11) showed two different LC phases before disordering into an isotropic liquid. Dendron-coil 10 displayed gyroid and lamellar LC phases with increasing temperature (Figure 6a), whereas dendron-coil 11 revealed A15 micellar cubic and hexagonal columnar LC phases (Figure 6b). Remarkably, a simple variation of dendron generation at a fixed linear PEO coil engineered a variety of LC phases with different $\mathrm{PEO}$ domain dimensionalities from zero, one and two up to three.

Besides the rich phase behavior, another notable feature of the dendron-coil assemblies is the temperature-dependent phase sequence: gyroid-to-lamellar and micellar-to-columnar for $\mathbf{1 0}$ and 11, respectively. These thermally induced phase sequences are unusual in comparison to linear block copolymers. ${ }^{21,26}$ The distinct assembling behavior must originate from the unique dendron-coil

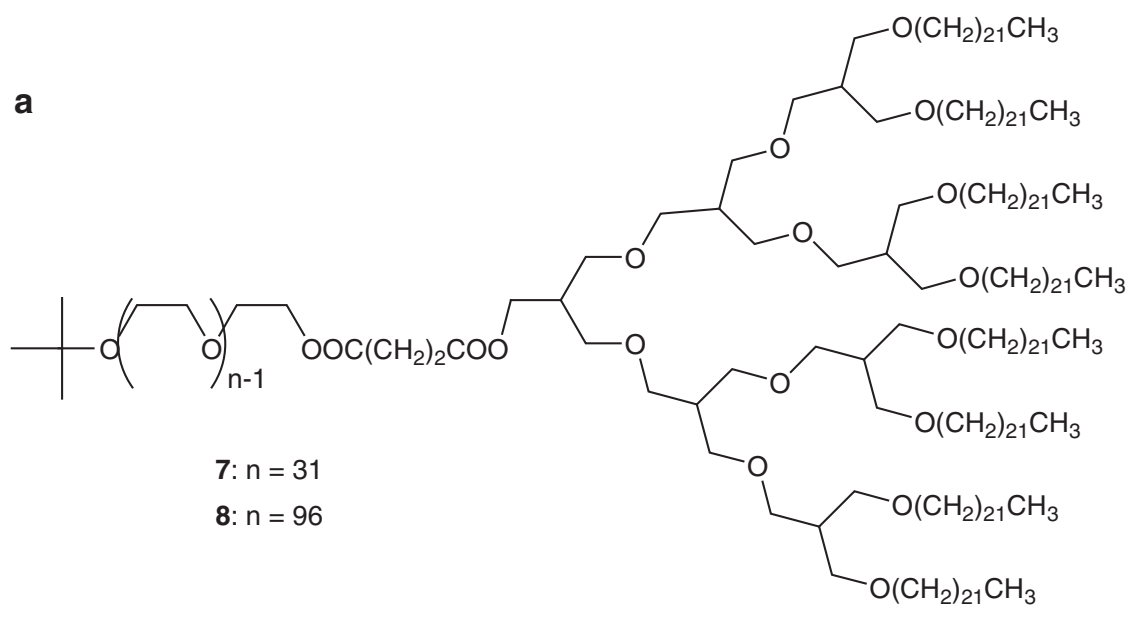

b

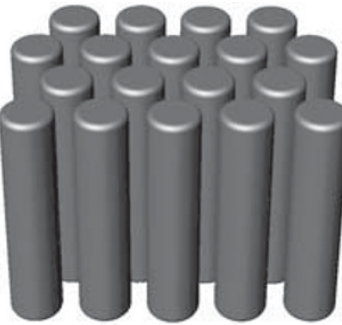

columnar

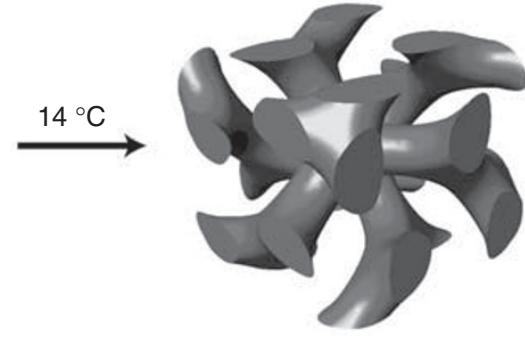

continuous cubic

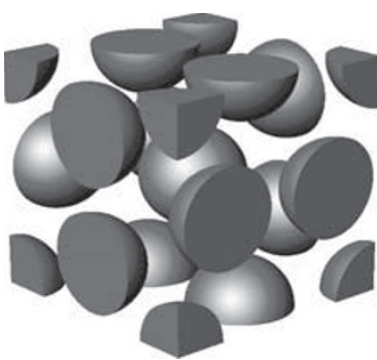

A15 micellar cubic
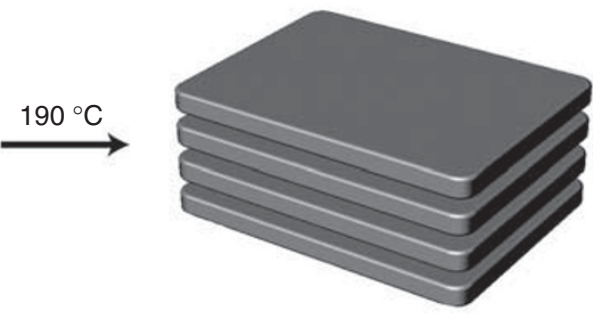

lamellar

Figure 3 (a) Molecular structures of dendron-coils $\mathbf{7}$ and $\mathbf{8}$, (b) the A15 micellar cubic liquid crystalline (LC) phase of $\mathbf{7}$ and (c) the LC phases of $\mathbf{8}$ as a function of temperature. For clarity, only hydrophilic domain structures are shown in $\mathbf{b}$ and $\mathbf{c}$. 
<smiles>CC(C)OCCC(C)(C)NC(=O)CCC(=O)NCC(CO)CO</smiles>

9: $R=R_{1}$

10: $R=R_{2}$

11: $R=R_{3}$

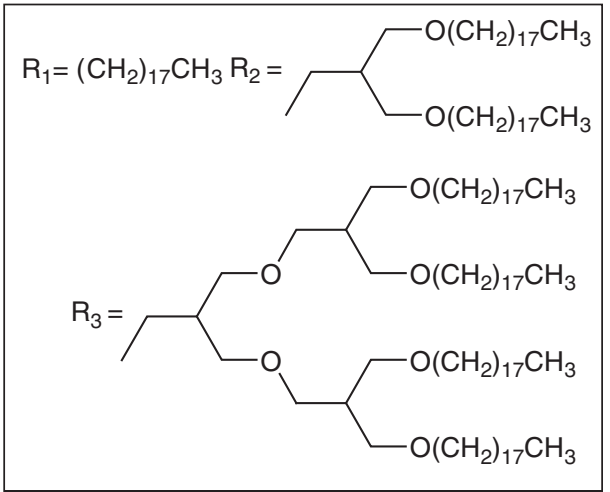

Figure 4 Molecular structures of dendron-coils 9,10 and 11 .

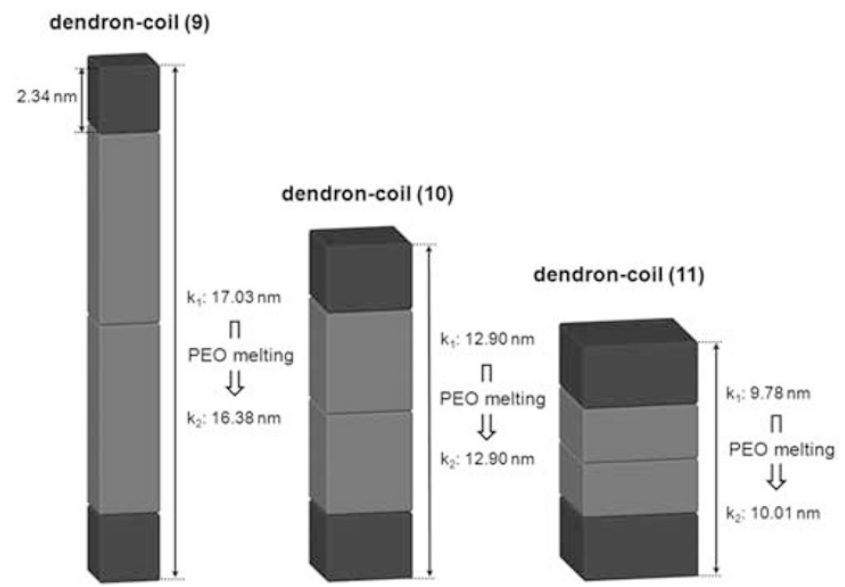

Figure 5 Molecular section variation of the dendron-coils in the bilayered crystalline phases. The dark and bright regions represent the hydrophobic and hydrophilic parts, respectively. $k_{1}$ : both alkyl and poly(ethylene oxide) (PEO) parts are crystalline, $k_{2}$ : the alkyl chain is crystalline, but the PEO coil is molten.

chain architecture. In contrast to linear block copolymers, the peripheral alkyl chains tend to locate the other matrix in order to reduce the steric hindrance, although its volume fraction is smaller than that of the linear PEO coil. In this configuration, the curved interface relaxes the peripheral octadecyl chains at the expense of an increased stretching penalty of the PEO chain. As the temperature increases, however, the conformational energy of the longer PEO coil in the inner core increases. Therefore, to alleviate the stretching penalty of the PEO coil with increasing temperature, the gyroid and micellar LC phases are transformed into the lamellar and columnar LC phases, respectively. Consequently, the simple molecular approach, a variation of the dendron generation at the fixed PEO coil length, can manipulate the assembling properties of the dendron-coil system in the solid and LC states.
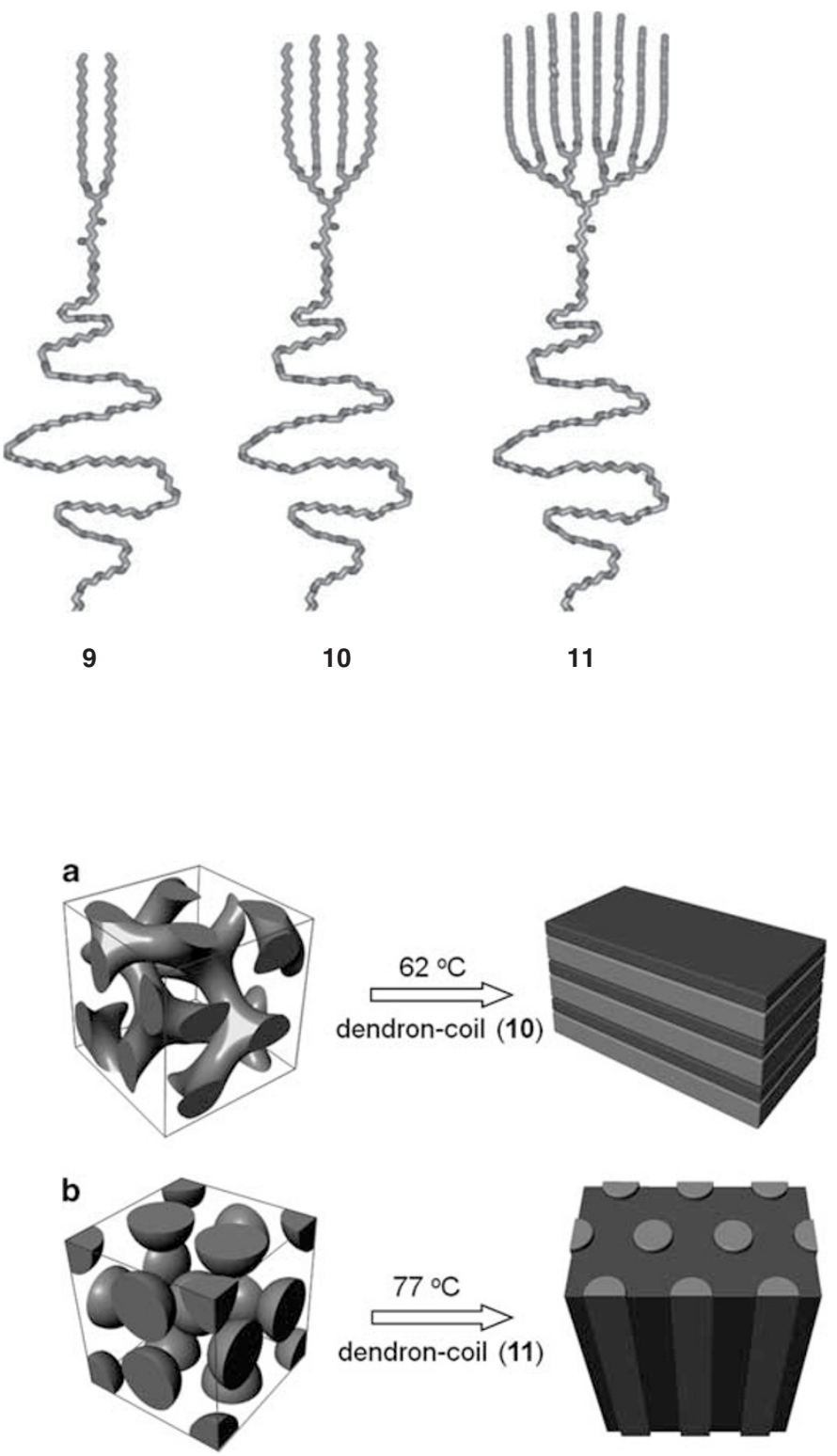

Figure 6 Liquid crystalline morphologies of dendron-coils 10 and $\mathbf{1 1}$. (a) Gyroid-to-lamellar transition of $\mathbf{1 0}$ and (b) micellar cubic-to-hexagonal columnar transition of $\mathbf{1 1}$. The dark and bright parts represent the hydrophobic and hydrophilic regions, respectively. For reasons of clarity, the hydrophobic matrix is omitted in the gyroid and micellar cubic phases.

\section{AMPHIPHILIC DENDRON-COIL-DENDRON TRIBLOCK COPOLYMERS BASED ON ALIPHATIC POLYETHER DENDRONS WITH SHORTER ALKYL PERIPHERIES}

The above-described dendron-coil examples are composed of long alkyl peripheries, which are docosyl and octadecyl chains for 7-8 and 9-11, respectively. Therefore, the alkyl crystallization strongly induced lamellar crystalline morphologies due to the enhanced molecular anisotropy. In contrast, the choice of shorter alkyl peripheries could lead to non-lamellar crystalline structures because of the reduced structural anisotropy. We applied this idea to dendron-coil-dendron triblock copolymers. ${ }^{28}$ Instead of the long alkyl chains, a shorter tetradecyl chain was chosen as the peripheral alkyl chain. The triblock block copolymers have two third-generation aliphatic polyether 


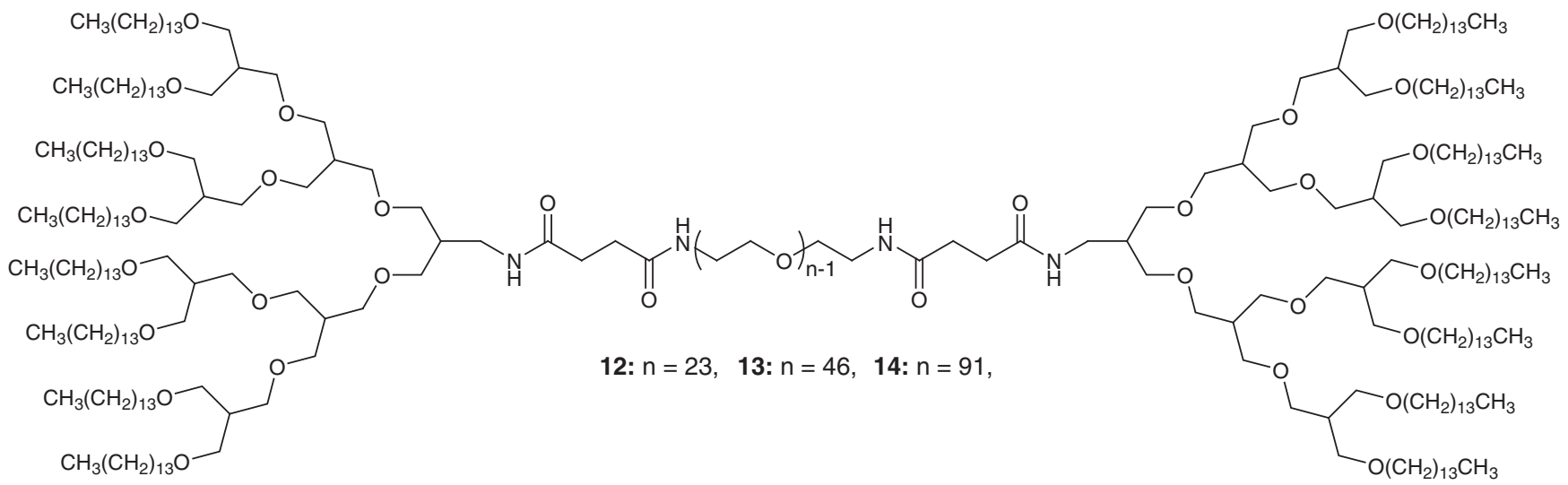

Figure 7 Molecular structures of dendron-coil-dendron triblock copolymers 12, 13 and 14.

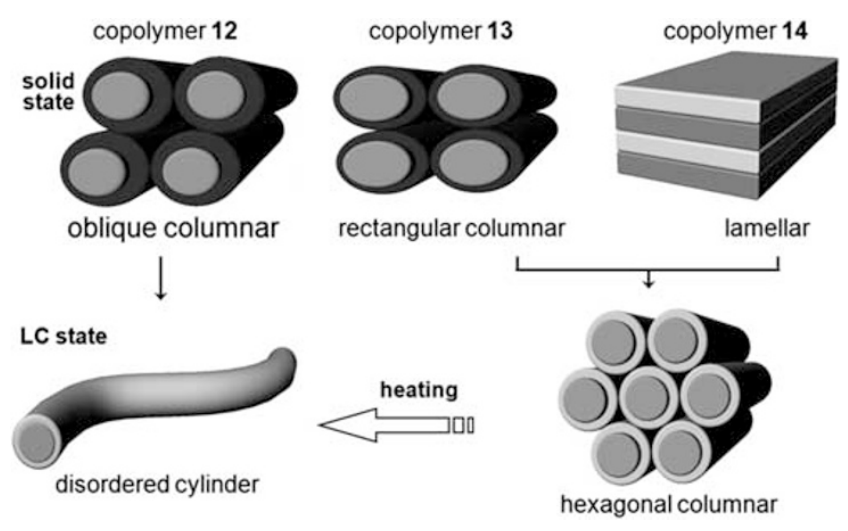

Figure 8 Assembled morphologies of copolymers 12, 13 and 14 in the solid and liquid crystalline (LC) states. Only alkyl chains are crystalline (darker region) in the oblique and rectangular columnar solid states, whereas only poly(ethylene oxide) (PEO) coils are crystalline (darker region) in the lamellar solid state. In the LC phases, the darker interior and lighter exterior consist of PEO coils and alkyl chains, respectively.

dendrons at both ends and a PEO middle, with different molecular weights (Figure 7). The triblock copolymers showed interesting thermal properties as a function of the middle PEO coil length. The copolymers (12 and 13) with the relatively shorter PEO coils $\left(M_{\mathrm{n}}=1000 \mathrm{~g} \mathrm{~mol}^{-1}\right.$ and $\left.2000 \mathrm{~g} \mathrm{~mol}^{-1}\right)$ revealed no PEO melting because of the large cross-section of the third-generation dendron ends and the amorphous PEO-like aliphatic polyether dendritic core. In contrast, copolymer 14 with the longest PEO coil $\left(M_{\mathrm{n}}=4000\right.$ $\mathrm{g} \mathrm{mol}^{-1}$ ) displayed PEO melting at $39.9^{\circ} \mathrm{C}$, which is even higher than that $\left(25.3^{\circ} \mathrm{C}\right)$ of tetradecyl peripheries. Interestingly, the tetradecyl crystallinity $(41 \%)$ in copolymer 14 is much higher than those (33 and 34\%) of copolymers 12 and $\mathbf{1 3}$ with the amorphous PEO coils. This might be attributed to the hard confinement effect by the crystalline PEO regions during the crystallization of the tetradecyl peripheries, which did not occur in copolymers 12 and $13 .{ }^{29}$

The self-assembling behavior in the solid states was complicated, depending on the PEO coil length (Figure 8). Copolymers 12 and 13, with amorphous $\mathrm{PEO}$ coils, self-assembled into oblique and rectangular columnar structures, respectively. In contrast, copolymer 14 with crystalline PEO coils exhibited a lamellar morphology. which is strongly driven by the crystallization of long PEO coils.
Moreover, all triblock copolymers exhibited microphase-separated structures in the LC state. On the basis of the X-ray scattering and transmission electron microscopy results, copolymer 12 exhibited a disordered cylindrical LC phase, which is different from a disordered micellar phase observed in linear block copolymers. ${ }^{30}$ The formation of the disordered cylindrical LC phase is probably due to the hydrogen bonding interaction between the amide groups linking the dendrons and middle PEO block. As the PEO coil length increased, copolymers 13 and 14 displayed an ordered columnar LC phase with a two-dimensional hexagonal symmetry as well as the disordered cylindrical LC phase. Consequently, complex thermal and assembling behaviors in the solid and LC states can be modulated by simple variation of the middle PEO coil length in the dendron-coil-dendron triblock copolymer system.

\section{IONIC TRANSPORTATION VIA LC SELF-ASSEMBLY OF DENDRITIC ASSEMBLERS}

The study of ionic transportation in ordered structures via selfassembly of liquid crystals or block copolymers is a particularly fascinating research topic for both academic and industrial applications because it may provide a novel approach for the invention of polymer electrolyte materials for secondary batteries and fuel cells. ${ }^{31}$

Dendritic assemblers have been used as nanostructured media for the study of ionic transportations. The Kato group reported diverse assembling dendritic systems based on Percec-type monodendron units. ${ }^{32-34}$ Their self-assembled ionic materials have mostly involved low-dimensional columnar structures as a supramolecular motif. For example, ionic monodendrons 15 and 16 bearing imidazolium salt at the focal group self-organized into a hexagonal columnar structure where the one-dimensional cylindrical core domain mediated ionic transportation (Figure 9a). ${ }^{35}$ In a multi-domain sample consisting of one-dimensional conducting cylinders, the ionic pathway is complicated, thereby exhibited low conductivity values. However, the Kato group overcame this drawback through a macroscopic orientation of conducting cylinders using a shear process (Figure 9b). The authors prepared two samples, which were aligned perpendicular and parallel to the electrodes, respectively, and examined the conductivity values. The sample whose columns are aligned 'perpendicular' to the electrodes showed 10 times higher ionic conductivity values than the sample whose columns are aligned 'parallel' to the electrodes. In addition, the polydomain samples without the shear process displayed conductivities between the values of the two differently aligned samples. 
a

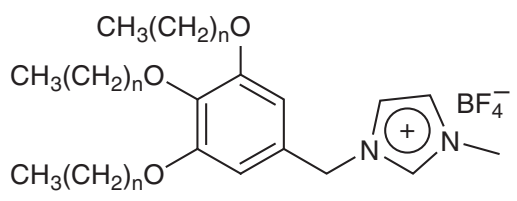

15: $\mathrm{n}=9$

16: $\mathrm{n}=11$ b

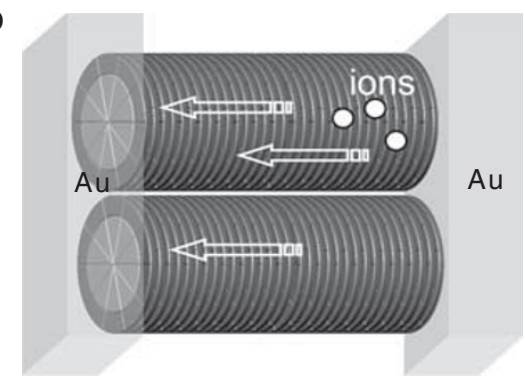

Figure 9 (a) Molecular structures of ionic monodendrons 15 and 16, and (b) the schematic of an oriented columnar sample after shearing the material in a perpendicular direction to the gold electrodes.

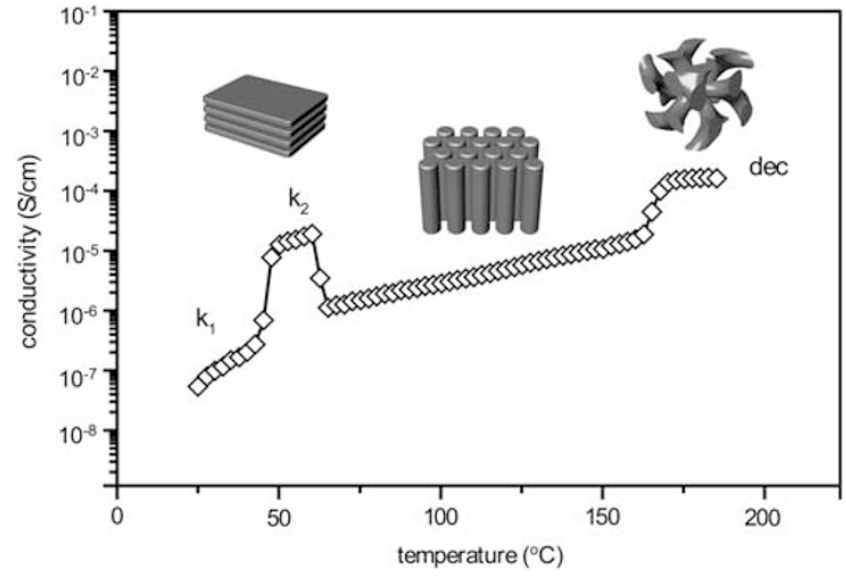

Figure 10 Conductivity data of the ion-doped dendron-coil $\left(\mathbf{8}-\mathbf{L i}^{+}\right)$as a function of temperature. k, crystalline; dec, decomposition.

Although the above result provides a useful way to advanced electrolyte materials, the alignment process cannot be applied to every assembling system. As an alternative method, a gyroid LC structure is particularly useful for polymer-electrolytes because it consists of intrinsically continuous conducting channels. In terms of structural connectivity, the gyroid morphology can offer an efficient threedimensional conducting path without macroscopic orientation.

Cho and Wiesner reported the first example showing ionic behavior in the gyroid LC structure. ${ }^{22}$ The authors demonstrated ionic transportion in the gyroid network structure using the dendroncoil sample $\left(\mathbf{8}-\mathbf{L i}^{+}\right)$doped with lithium triflate. The ion-doped sample with $\left[\mathrm{Li}^{+}\right] /[\mathrm{EO}]=0.02$ exhibited lamellar, hexagonal columnar and gyroid LC phases as a function of temperature (Figure 10). By tracking the conductivities on heating, a direct comparison of the conducting properties between the gyroid and the other morphlogies could be presented. In contrast to a gradual conductivity change in conventional PEO-based electrolytes, the conductivity data showed decrease and rebound associated with the phase transitions (Figure 10). Among the observed phases, the gyroid LC phase exhibited the highest conductivity. This is because the gyroid LC structure is composed of hydrophilic networked channels, which enable the three-dimensional ionic transport. In contrast, the lamellar and columnar structures are composed of discontinuous two-dimensional layers and one-dimensional cylinders, respectively, thus in the multi-domain sample the ionic courses are more complicated than the gyroid structure. Consequently, the authors concluded that the ion-conducting behavior is strongly correlated to the dimensionality of the PEO domain, and the three-dimensional connectivity of the PEO domains in the gyroid resulted in the enhanced ion-transporting phenomenon.

The Kato group also reported a gyroid ionic channel formation via self-assembly of fan-shaped dendritic molecules 17 and 18 (Figure 11a). ${ }^{36}$ The molecules contain ammonium salt at the focal point. Interestingly, the dendritic molecules with decyl and dodecyl peripheries formed gyroid structures at room temperature. Similar to the example by Cho and Wiesner, in comparison with the nonoriented columnar structure at higher temperatures, the room temperature gyroid structures exhibited higher conductivities. This result can also be explained by the three-dimensional continuous channel structure (Figure 11b).

\section{ION-CONDUCTING GYROID PHASES IN DENDRITIC SYSTEMS CONTAINING SMALL ADDITIVES}

In some cases, the described esthetic gyroid structure could be obtained by blending dendritic assemblers with small hydrophilic additives. As well as gyroid formation, the insertion of hydrophilic small additives has another merit in ionic transportation because the low viscosity of additives facilitates the movement of ions in conducting regions.

The Gin group reported crosslinked polymeric materials by blending a monomeric minidendron with a $0.245 \mathrm{M} \mathrm{LiClO}_{4}$-propylene carbonate (PC) solution, and subsequently photo-polymerizing the composite sample. ${ }^{37}$ The monomeric minidendron (19) contains lithium 2-aminoethanesulfonate at the focal site, which resulted in the core part being compatible with the electolyte solution (Figure 12). The electrolyte sample has $15 \mathrm{wt} \% 0.245 \mathrm{M} \mathrm{LiClO}_{4}$-PC solution. The electroyte composite showed a gyroid-like continuous morphology at room temperature. Even after a photo crosslinking, the morphology was retained, as confirmed by optical polarized microscopy and X-ray diffraction. The polymerized films were flexible and mechanically robust. The measured conductivity at room temperature was $9 \times 10^{-4} \mathrm{~S} \mathrm{~cm}^{-1}$, which is comparable to that of gelled PEO systems. ${ }^{38}$ The magnitude of the conductivity values indicates a liquid-like mobility in the ionic channels.

Recently, we reported a correlation between the conducting and LC properties in a mixture system consisting of a dendron-coil (20) and a lithium-doped hydrophilic additive (21; Figure 13). ${ }^{39}$ In this work, we used a dibranched hydrophilic molecule consisting of two tri(ethylene oxide) coils. The additive is nonvolatile and noncrystalline, which could be useful for electrolyte applications. The dendron-coil (20) is composed of a hydrophobic Y-shaped dendron block and a hydrophilic linear PEO coil with $M_{\mathrm{n}}=750 \mathrm{~g} \mathrm{~mol}^{-1}$, and exhibited a hexagonal columnar LC phase. The synthesis of the dendron-coil used stepwise 'click' reactions. The advantages of the click reacton in the 
a

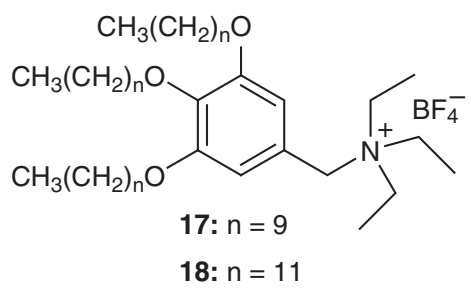

b

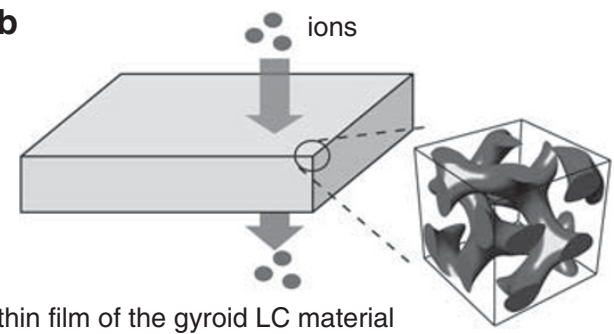

Figure 11 (a) Molecular structures of 17 and 18, and (b) a three-dimensional ion channel network based on a gyroid structure. LC, liquid crystalline.

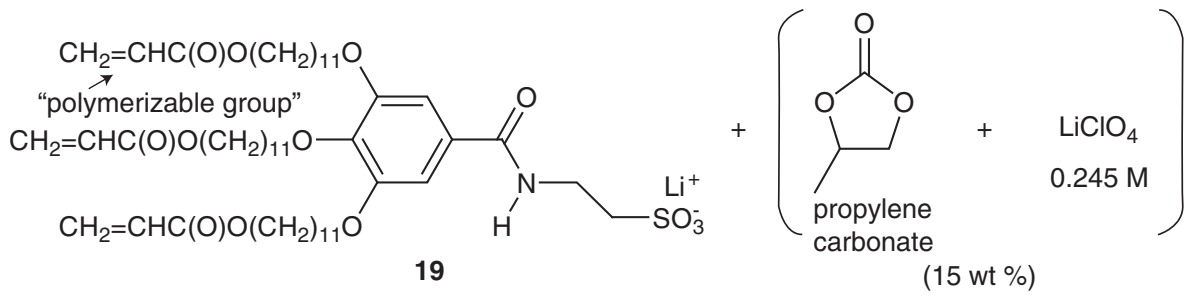

Figure 12 Li ion-conducting solid-liquid composite showing a gyroid-like channel structure.

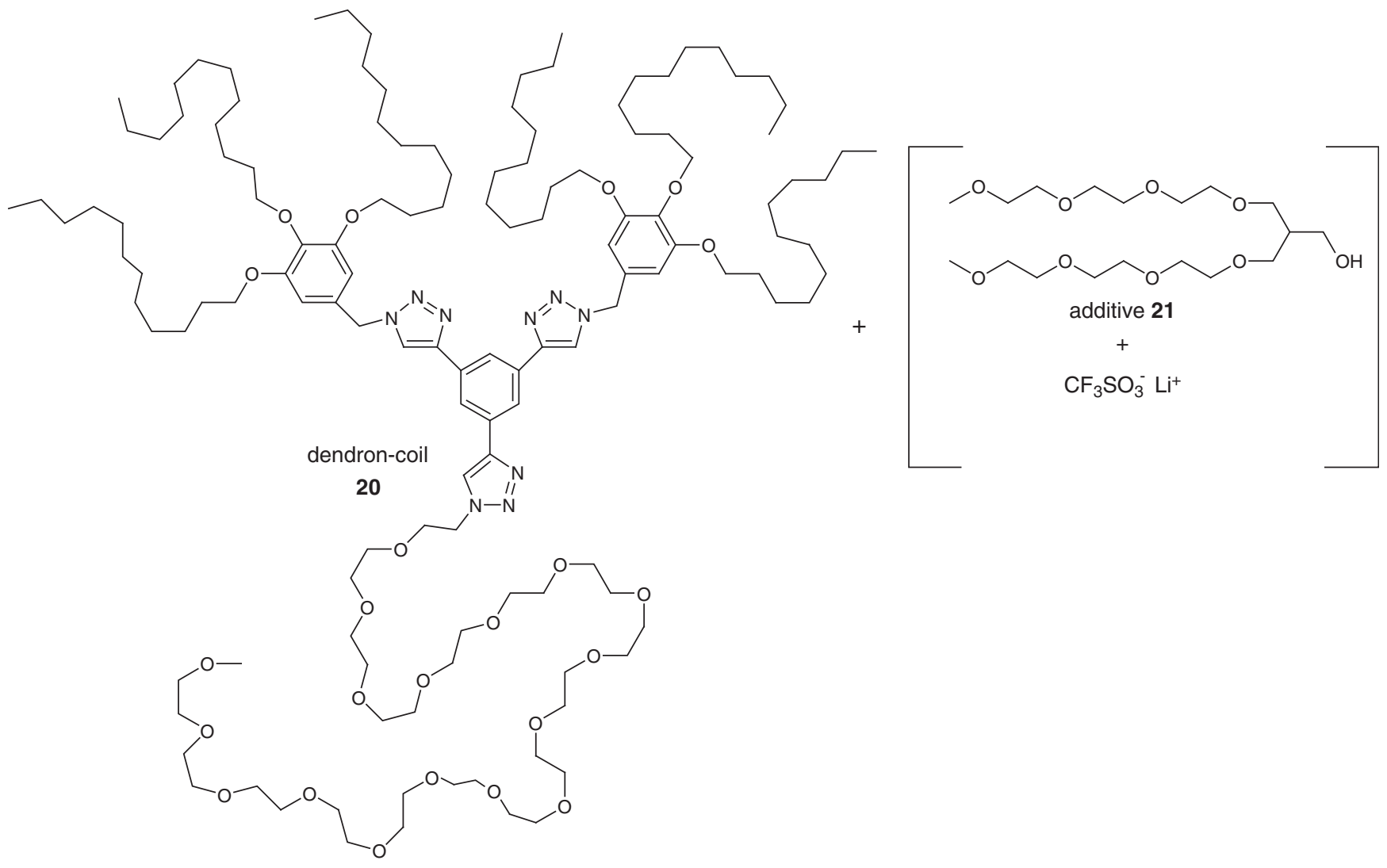

Figure 13. Molecular structures of dendron-coil $\mathbf{2 0}$ and additive 21 .

preparation of self-assemblers are explained in a latter part of this article. By adding an additive mixture bearing lithium triflate salt $\left(\left[\mathrm{Li}^{+}\right] /[\mathrm{EO}]=0.05\right)$, a series of ionic samples with the additive weight fractions $\left(f_{\mathrm{w}}\right)$ of 10,20 and $30 \%$ were prepared.
In contrast to the columnar LC phase of the prisine dendron-coil (20), a biphasic phenomenon displaying both columnar and gyroid LC phases was observed in the ionic samples with $f_{\mathrm{w}}=10$ and $20 \%$. By increasing the amount of the additive mixture, the gyroid LC 
phase became dominant, and finally only a gyroid phase was exhibited in the sample with $f_{\mathrm{w}}=30 \%$. Ionic conductivity in the biphasic system reflects the sum of the ion-transporting properties in both phases. The ionic conductivity increased with increasing the portion of the gyroid structure (Figure 14). As the gyroid portion increased, the conductivity value increased from $3.80 \times 10^{-6}$ to $2.34 \times 10^{-5} \mathrm{~S} \mathrm{~cm}^{-1}$ at $35^{\circ} \mathrm{C}$. Consequently, the conductivity value increased $\sim 2.5$ times with each addition. The conductivity growth could be explained by the interplay of the high mobility of the small additive and the morphological transformation from an onedimensional to three-dimensional form of the ion-conducting cylindrical cores.

\section{ION TRANSPORT IN THE CONTINUOUS MATRIX OF THE ONE-DIMENSIONAL COLUMNAR LC STRUCTURE OF DENDRON-COILS}

As mentioned above, in order to enhance the ion-transporting behavior, one-dimensional columnar LC structures have to be macroscopically aligned. However, it is still challenging to apply the

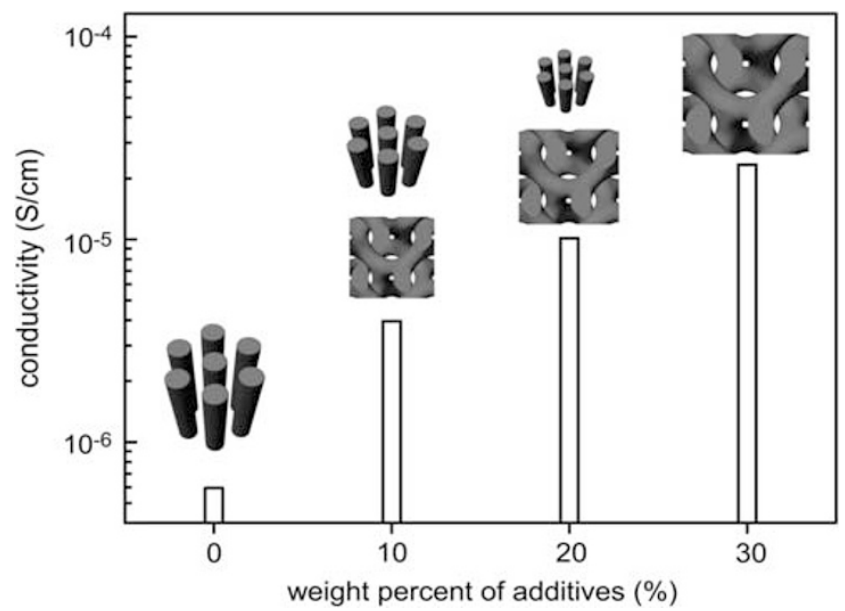

Figure 14 Conductivity data as a function of the weight percent of additives at $35^{\circ} \mathrm{C}$. orientation process to other molecular systems. An alternative approach is to use the gyroid structure bearing three-dimensional conducting channels. Despite the versatile three-dimensional continuous channel structure, the morphology is still difficult to develop at ambient temperature, and in some cases disappears upon ion doping. ${ }^{40}$

As another way for enhancing ion transport in nanostructured LC phases, we recently suggested the use of a continuous matrix region of a columnar phase as an ion-conducting phase. ${ }^{41}$ The merit in this approach is to readily achieve a three-dimensional conducting phase from conventional columnar structures. To locate hydrophilic PEO coils in the matrix, we devised hydrophilic PEO-like dendrons via stepwise convergent synthesis. ${ }^{42}$ The hydrophilic third-generation dendron was covalently combined with a hydrophobic polystyrene (PS) coil, producing a dendron-coil block copolymer (22) (Figure 15).

Before doping lithium salt, the block copolymer showed no microphase-separated structure as characterized by the differential scanning calorimetry and small-angle X-ray scattering data. However, two lithium-doped samples with different lithium concentrations exhibited two glass transitions corresponding to dendritic PEO and PS, respectively. This indicates that an ion-doped PEO block is incompatible with the PS block due to the increased immiscibility on lithium doping. The ionic sample (22-0.21) with $\left[\mathrm{Li}^{+}\right] /[\mathrm{EO}]=0.21$ displayed a lamellar structure, whereas the other ionic sample (22-0.42) with $\left[\mathrm{Li}^{+}\right] /[\mathrm{EO}]=0.42$ exhibited a hexagonal columnar structure.

Interestingly, the conductivity of the columnar sample was higher than that of the lamellar sample by two orders of magnitude (Figure 16a). Considering that the optimum lithium ion concentration was found to be about 0.05 for typical PEO-based electrolytes, the observed conductivity result is attributed to the structural features of the morphologies. The lamellar sample consists of discrete twodimensional PEO-lithium salt sheets. On the other hand, the columnar sample is composed of a continuous hydrophilic region, which can lead to three-dimensional transportation of lithium ions (Figure 16b). In addition to the conductivity properties, the columnar sample (22-0.42) is superior to the lamellar sample (22-0.21) in terms of mechanical strength. The elastic moduli $\left(\mathrm{G}^{\prime}>10^{4} \mathrm{~Pa}\right)$ of the columnar sample were found to be higher than those of the lamellar

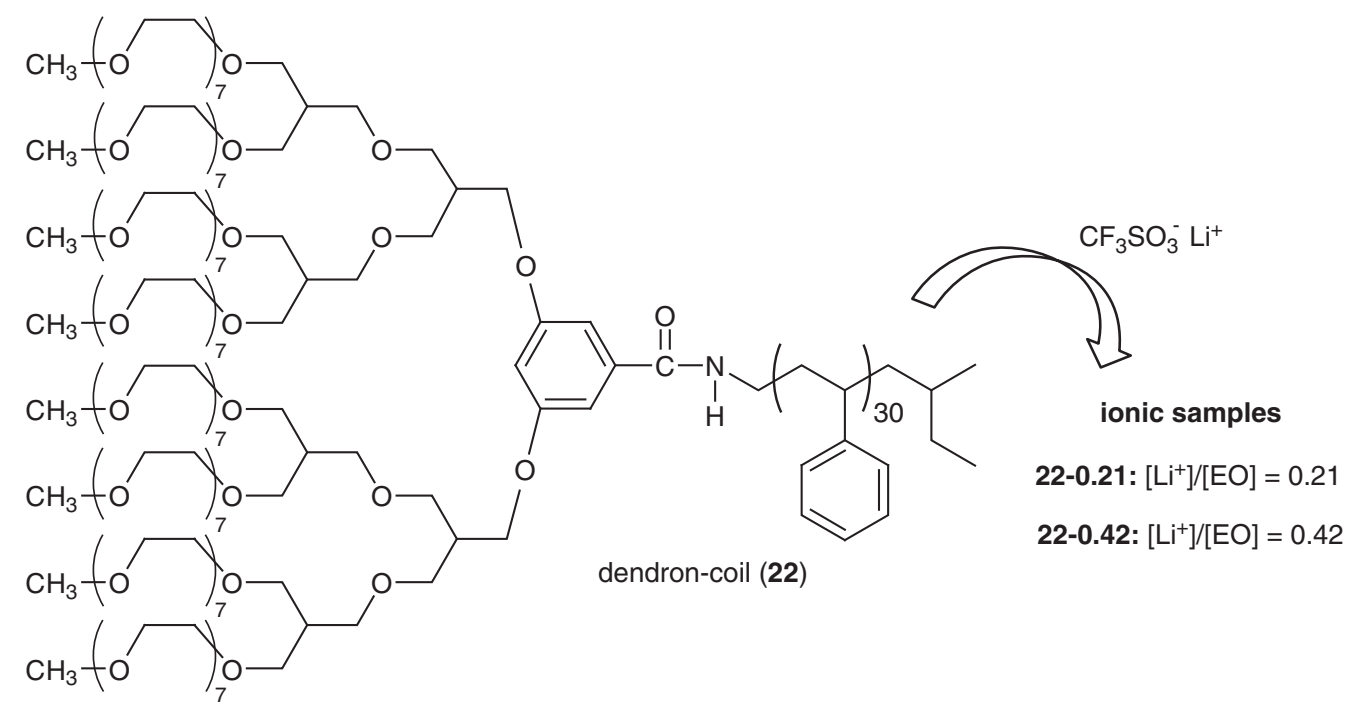

Figure 15 Molecular structure of dendron-coil 22 and ionic samples doped with lithium triflate. 


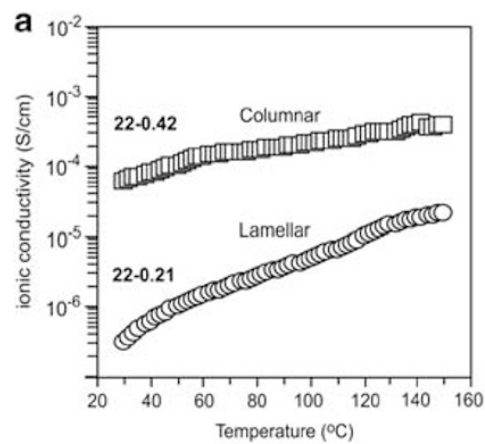

b

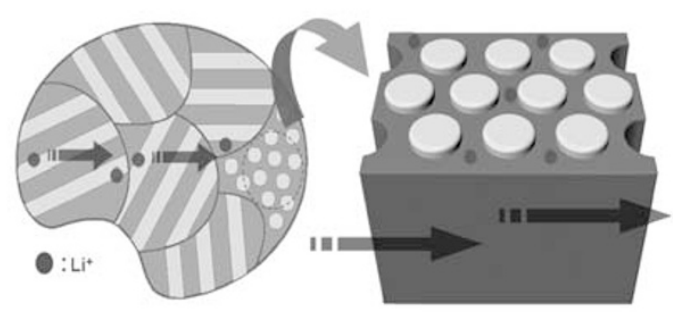

Figure 16 (a) Ionic conductivities and (b) schematic sketch of continuous ionic transportation in the matrix of the multi-domain columnar assembly of 22-0.42 (dark gray, continuous poly(ethylene oxide) matrix; bright gray, cylindrical PS cores).

sample. $^{21}$ Consequently, the columnar ionic sample with the continuous ionic matrix is more versatile than the lamellar one with respect to ionic transportation as well as mechanical strength.

\section{BLOCK CODENDRIMER ASSEMBLIES}

A new dendritic assembling system of interest is block codendrimers, where two constitutively different dendrons are covalently bound at the center. This molecular system has had little investigation in comparison to other block copolymer systems. Block codendrimers are a different class of self-assembling system from linear block copolymers and dendron-coil hybrids. So far, several block codendrimers have been reported, and some of them displayed LC properties in melt. These mostly contain mesogenic dendrons, for example, Percec-type poly(benzyl ether) dendrons, as their basic building unit. ${ }^{43-45}$ On the other hand, despite several reports suggesting the synthesis of block codendrimers carrying nonmesogenic dendrons, ${ }^{46-48}$ only a few self-assembling codendrimers have been reported, ${ }^{49,50}$ and only a lamellar morphology has been found in the melt.

To obtain a rich LC phase behavior, we devised a block codendrimer system consisting of two different aliphatic polyether dendrons whose peripheries were hydrophilic tri(ethylene oxide) and hydrophobic tetradecyl chains (Figure 17). ${ }^{51}$ As another structural uniqueness of these codendrimers, they contain an aromatic disk that was prepared by stepwise click reactions. Thus, the codendrimers are referred to as discotic block codendrimers. No LC phase was observed in any of the block codendrimers because the degree of mixing between the hydrophilic and hydrophobic blocks in the dendrimer architecture is greater than that in linear block copolymers. This interpretation is consistent with recent theoretical work by Pickett and Rios, which predicted that it is difficult to obtain a segregated regime in block codendrimers. ${ }^{52}$ However, ion doping in the PEO block induced LC phases in all ion-doped samples, which is due to increased immiscibility. ${ }^{53}$

The block codendrimer (23) with two hydrophilic dendrons showed gyroid and hexagonal columnar LC phases, as lithium concentration and/or temperature increased (Figure 18a). The gyroid LC phase (typically observed in linear block copolymers) in this study is the first example of dendrimer-architectured self-assemblers. In addition, the columnar LC phases exhibited a fan-like texture from the optical polarized microscopic investigation, which suggests the anisotropic packing of aromatic disks in the LC phase. ${ }^{54}$ On the basis of the morphological and optical properties, our discotic block codendrimers are a novel class of a self-assembling system, which shares the morphological characteristic of block copolymers and

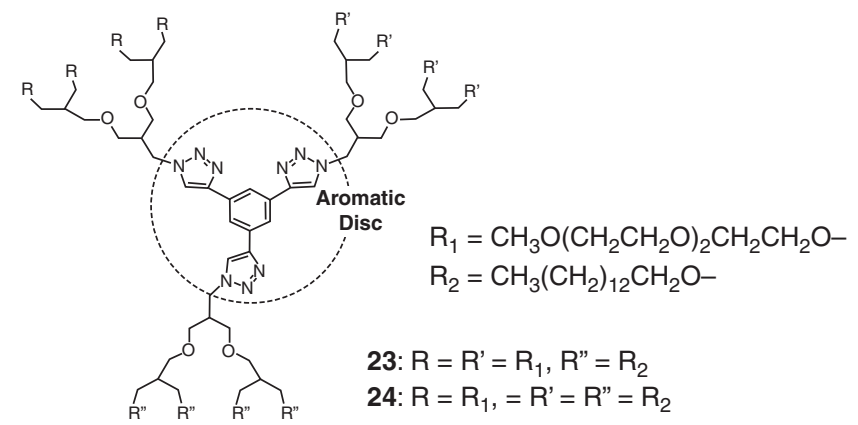

Figure 17 Molecular structures of discotic block codendrimers 23 and 24.

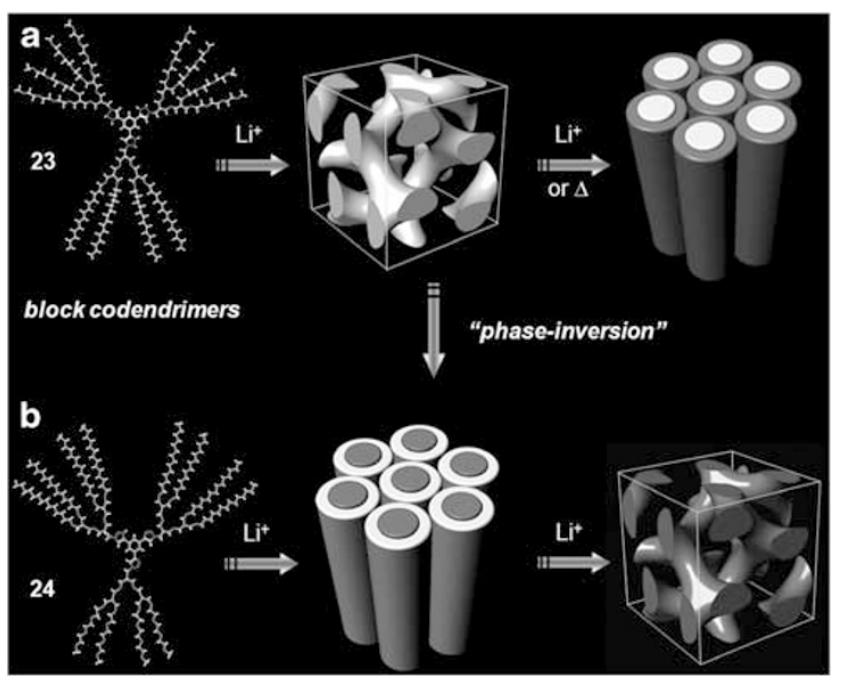

Figure 18 Gyroid and hexagonal columnar LC phases of block codendrimers 23 and 24. Bright and dark gray regions in the schematics represent the hydrophobic and hydrophilic parts, respectively.

discotic LC molecules. In contrast, the other codendrimer (24) with one hydrophilic dendron exhibited inverted-hexagonal columnar and gyroid LC phases with increasing lithium concentration (Figure 18b). Unlike the LC phases of the prior dendrimer samples, the columnar and gyroid structures in these samples from codendrimer 24 consist of hydrophobic matrixes and hydrophilic cylindrical cores. Consequently, along with the generation of a unique gyroid LC phase, the 


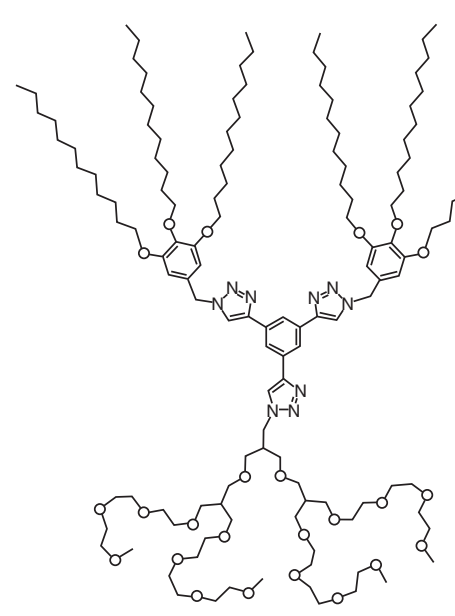

block codendrimer (25)

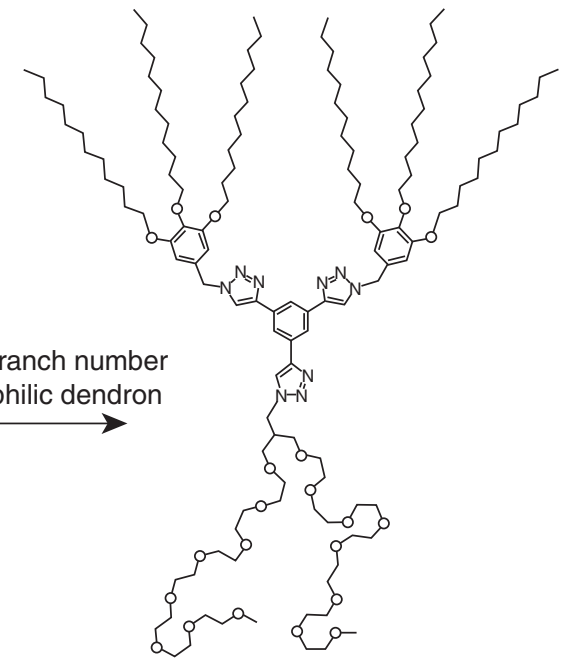

block codendrimer (26)

Figure 19 Molecular structures of block codendrimers $\mathbf{2 5}$ and $\mathbf{2 6}$ with tetrabranched and dibranched hydrophilic dendrons, respectively.

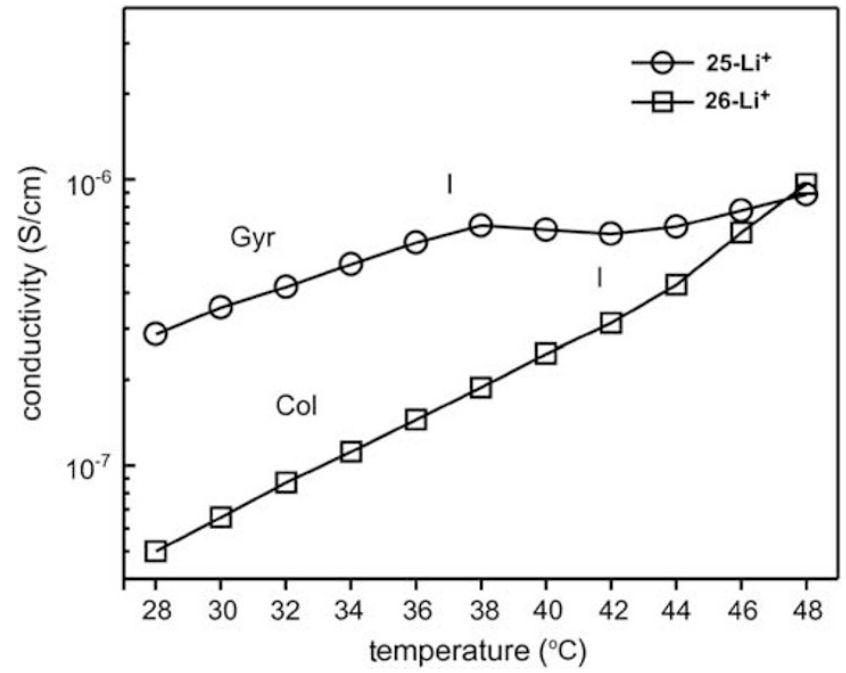

Figure 20 Ionic conductivities of ionic samples $25-\mathrm{Li}^{+}$and $26-\mathrm{Li}^{+}$ depending on the self-assembled morphology. Gyr, gyroid; Col, hexagonal columnar liquid crystalline phases; I, isotropic liquid.

local properties in the gyroid and columnar morphologies (that is, the inversion of hydrophilic and hydrophobic properties between cylindrical cores and matrix) could be fine-tuned; in other words, we can endow an exterior matrix with either a hydrophilic or hydrophobic character by the simple alteration of the majority dendritic block (Figure 18).

As a next, we attempted to engineer block codendrimer assemblies by simply varying the degree of chain branching in the hydrophilic dendron structure. ${ }^{55}$ We prepared two Janus dendrimers 25 and 26 consisting of hydrophobic Percec-type dendron and hydrophilic aliphatic polyether dendrons (Figure 19). The two dendrimers have almost identical molecular weights, but their degrees of branching in the hydrophilic dendron were designed to be dibranched and tetrabranched for $\mathbf{2 5}$ and $\mathbf{2 6}$, respectively. Similar to the previous examples, the pristine Janus dendrimers displayed no LC property, but upon doping lithium salt $\left(\left[\mathrm{Li}^{+}\right] /[\mathrm{EO}]=0.05\right)$, LC phases were induced in both samples. The ionic sample from 25 showed a gyroid LC phase, but the other ionic sample from $\mathbf{2 6}$ formed a hexagonal columnar LC phase. The distinct LC properties could be interpreted to be associated with the degree of branching in the hydrophilic PEO dendron. For the tetrabranched dendron, the higher grafting density of the PEO branches leads to greater lateral expansion, which resulted in the gyroid structure.

After the morphological analysis in the LC phases, their morphologydependent ionic conductivities were examined (Figure 20). The gyroid sample exhibited $\sim 5.4$ times higher values than the columnar sample. In addition, on entering the structureless liquid phases, the conductivity values of both ionic samples gradually merged. As mentioned earlier, this result reflects the efficient ion delivery of the gyroid LC structure consisting of three-dimensional hydrophilic networked channels.

\section{POLY(BENZYL ETHER) DENDRON-BASED DISCOTIC LC MATERIALS USING 'CLICK CHEMISTRY'}

Click chemistry, that is, a copper-catalyzed $[2+3]$ cycloaddition reaction between organic azides and alkynes, has been increasingly used in various chemistry fields, because of its outstanding merits such as high conversion and no side product. ${ }^{56}$ This click chemistry could be a powerful tool for the design of LC molecules, because the formed heterocyclic 1,2,3-triazole has a planar structure and high chemical/thermal stability. Thus, by direct conjugation with other aromatic rings, a molecular anisotropy (a prerequisite for LC formation) can be achieved. Nevertheless, only a few LC molecules created through the use of click chemistry have been reported, and all of them were calamitic molecules showing smectic or nematic LC phases. ${ }^{57-59}$ Therefore, it is interesting to devise discotic LC materials via the click chemistry.

Our group reported for the first time discotic LC molecules 27 -29 using an efficient click reaction. ${ }^{60}$ By doing a single click reaction of azido-functionalized Percec-type dendrons with 1,3,5-triethynylbenzene, the extension to a conjugate discotic mesogen consisting of three 1,2,3-triazoles and a central benzene ring was easily achieved (Figure 21).

The LC phase behavior was investigated as a function of peripheral alkyl chain length. As the peripheral alkyl chain was elongated, the 


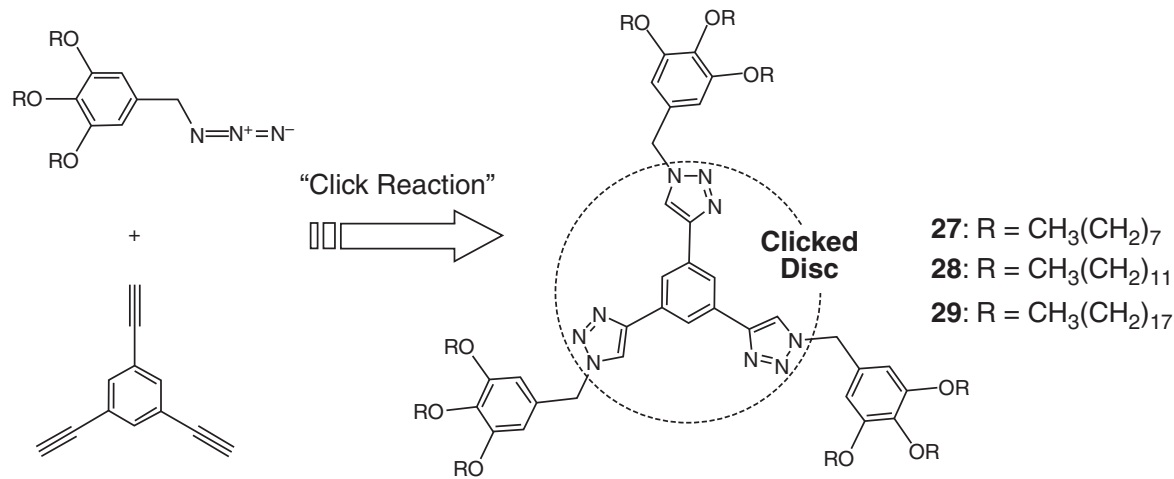

Figure 21 Design concept via click chemistry, and molecular structures of 27-29 with the extended triazole-based disk.
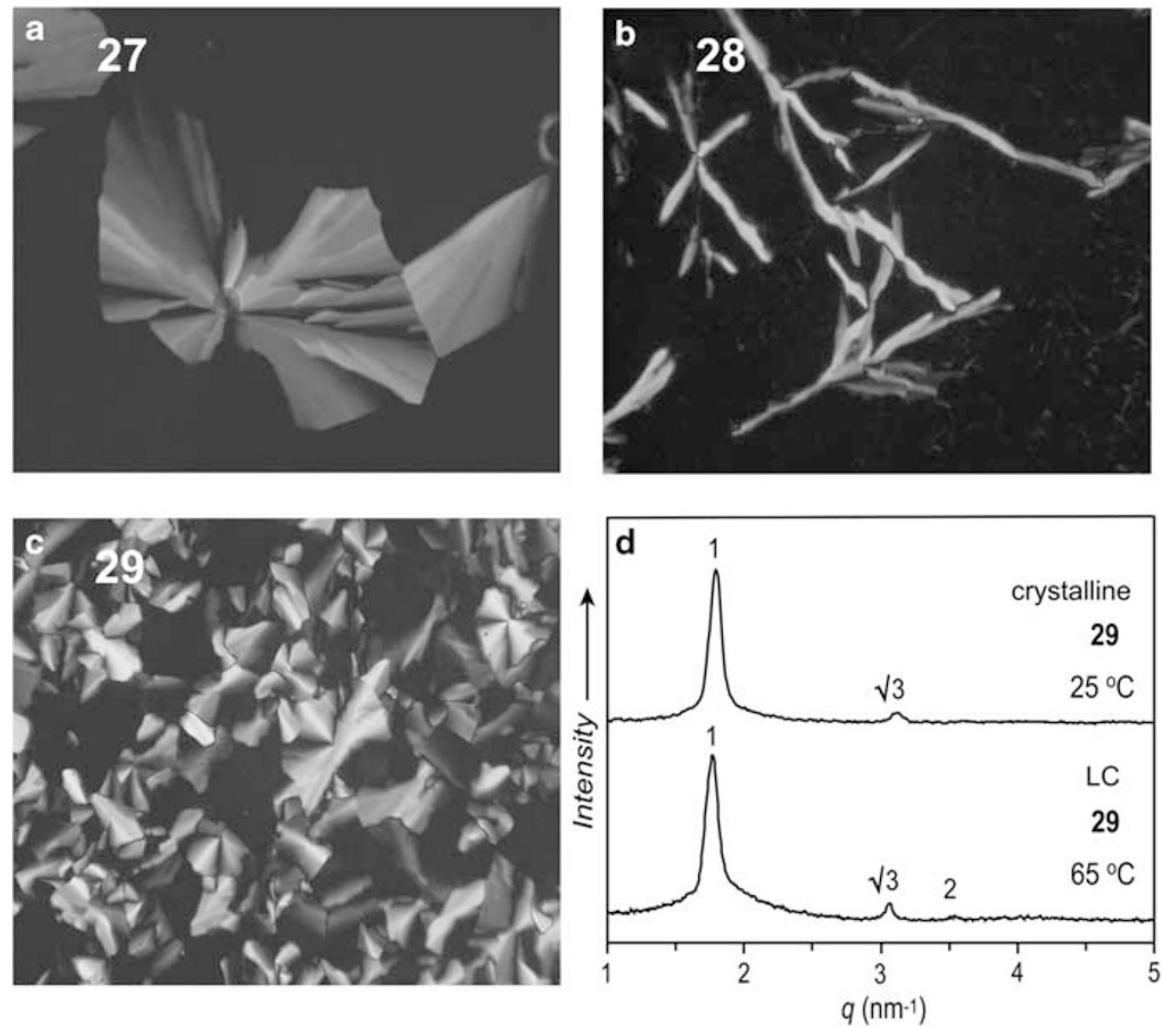

Figure 22 Optical textures of (a) 27 at $80^{\circ} \mathrm{C}$, (b) 28 at $70^{\circ} \mathrm{C}$, (c) 29 at $70^{\circ} \mathrm{C}$ and (d) the hexagonal columnar small-angle X-ray scattering patterns of 29 in the crystalline and liquid crystalline (LC) phases.

isotropization temperature decreased, while the crystal melting temperature increased. Discotic molecules 27-29 exhibited stable LC phases on both heating and cooling scans. The observed LC phases were revealed as two-dimensional hexagonal columnar structures. Interestingly, all showed a strong tendency to align homeotropically on the glass substrate, which may be potentially utilized for pattern applications on distinctly chemically modified surfaces (Figure $22 \mathrm{a}-\mathrm{c}){ }^{61,62}$ Unlike 27 and 28 , the crystalline phase of 29 was experimentally accessible. Interestingly, the crystalline phase maintained the two-dimensional hexagonal columnar structure similar to its LC phase, as confirmed by the small-angle X-ray scattering data (Figure 22d).

In addition to the above classical covalent approach, a supramolecular method can be utilized to prepare discotic LC materials. ${ }^{63-65}$
Interestingly, the triazole group produced after a click reaction can be a hydrogen-bonding motif, because the triazolyl hydrogen and nitrogen can be a hydrogen donor and acceptor, respectively. ${ }^{66}$ Thus, we attempted for the first time to use clicked triazolyl moieties as a hydrogen-bonding motif for the construction of discotic LC materials. ${ }^{67}$ In this work, we prepared a series of supramolecular discogens (HBC-n) via complementary hydrogen bondings between clicked $C_{3}$-symmetry triazole derivatives (TTB- $\boldsymbol{n}$ ) and 3,4,5-trialkoxybenzene carboxylic acids (TBA) (Figure 23). The simulation analysis suggested some amount of energy gains when forming the hydrogen-bonded complex. Indeed, several spectroscopic results, including infrared, proton nuclear magnetic resonance and emission data, confirmed the involvement of triazolyl hydrogen and nitrogen as well as central benzenyl hydrogen in the complex formation. 

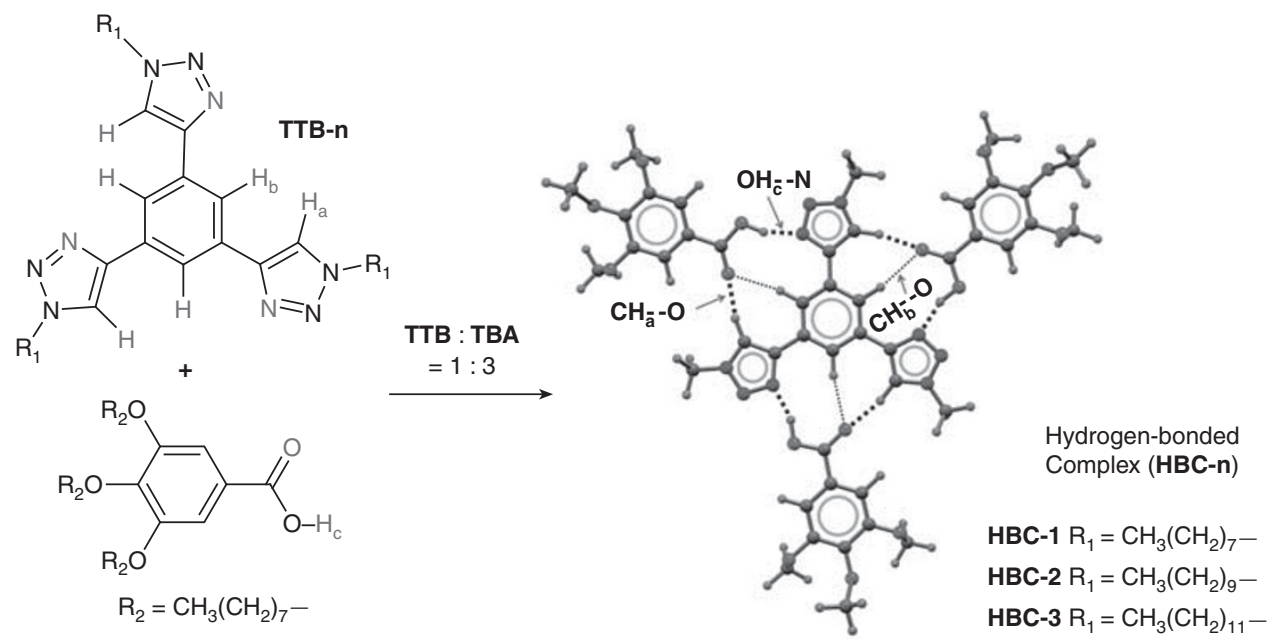

Figure 23 Molecular structures of TTB- $\boldsymbol{n}$ and trialkoxybenzene carboxylic acid (TBA), and the optimized geometry of HBC via complementary H-bondings.
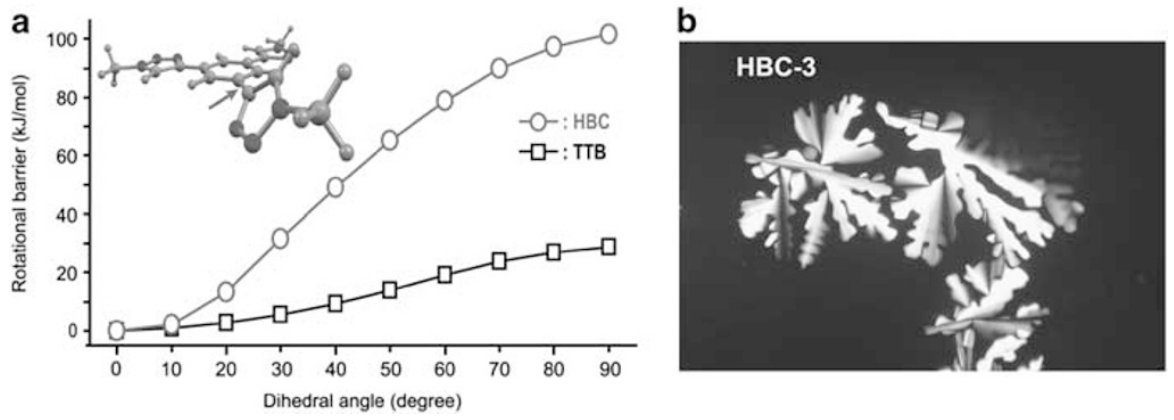

Figure 24 (a) Rotational energy barriers of TTB and HBC as a function of the dihedral angle with respect to the C-C bond (arrow) and (b) the dendritic optical liquid crystalline texture of HBC-3.

To see whether the hydrogen-bonded complex can act as a discotic mesogen, a preliminary simulation was examined. As a result, the rotational barrier of the $\mathrm{C}-\mathrm{C}$ bond linking the central benzene to the triazolyl ring was found to increase by the multiple hydrogen bondings with the carboxylic acids (Figure 24a). This indicates that the hydrogen-bonded discotic complexes have an extended mesogenic disk. As confirmed by the small-angle X-ray scattering and optical polarized microscopic observations, all the discotic complexes showed a monotropic hexagonal columnar LC phase (Figure 24b). Consequently, we demonstrated that the clicked triazolyl group can be utilized as a complementary hydrogen-bonding motif for the construction of discotic LC materials.

\section{CONCLUSIONS}

Self-assemblers with hybrid-chain architectures can provide LC assemblies in the bulk state. In this article, we reviewed our recent hybrid assemblers, such as dendron-coils, dendron-coil-dendrons, block codendrimers and discotic LC materials. In particular, welldefined aliphatic polyether dendrons, as well as Percec-type monodendrons, have been utilized as a constituting branched building unit. Because of their high thermal and chemical stability, the aliphatic polyether dendrons could be a useful candidate for designing polymeric materials. The dendron-coil system including the diblock and triblock copolymers showed different morphological properties in comparison with conventional linear block copolymers. In addition, newly designed block codendrimers consisting of hydrophilic and hydrophobic dendrons exhibited a unique assembling feature on ion doping. The observed morphological feature shares the characteristics of discotic LC and block copolymers, on the basis of the optical texture of the columnar LC phase and the observed gyroid LC phase. In addition to the morphological properties, the ordered LC phases in monodendrons, dendron-coils and block codendrimers could be used as ionic media responsible for charge transportations.

The dendritic building units could be applied to the design of discotic LC materials. We prepared discotic LC molecules based on the Percec-type monodendrons using an efficient click chemistry. All the discotic molecules showed homeotropic columnar LC phases where cylinders are aligned perpendicular to the glass substrate. Discotic LC materials can also be prepared via complementary hydrogen bondings between $C_{3}$-symmetric clicked discogens and a Percec-type monodendron with a carboxylic acid focal group. The simulation and experimental results proved that the hydrogen-bonded complexes formed hexagonal columnar LC phases.

Hybrid molecular systems based on well-defined dendrons can provide a variety of LC phases with tunable local properties, and these LC morphologies can be used as a structural platform for the development of advanced functional materials. Although this review paper mainly deals with the bulk assemblies and applications of molecular assemblers based on the dendritic building blocks, many of assemblers mentioned in this paper consist of hydrophilic and 
hydrophobic components. Because of their amphiphilic character, their solution assembling behavior may be another interesting research topic, which can be applicable to nanostructured drug delivery and sensory materials. Thus, the hybrid assemblers via rational molecular tailoring may offer novel playgrounds for soft functional materials.

1 Lehn, J. M. Supramolecular Chemistry (VCH, Germany, 1995).

2 Kato, T., Mizoshita, N. \& Kishimoto, K. Functional liquid-crystalline assemblies: selforganized soft materials. Angew. Chem. Int. Ed. 45, 38-68 (2006).

3 Goodby, J. W., Saez, I. M., Cowling, S. J., Görtz, V., Draper, M., Hall, A. W., Sia, S. Cosquer, G., Lee, S. -E. \& Raynes, E. P. Transmission and amplification of information and properties in nanostructured liquid crystals. Angew. Chem. Int. Ed. 47, 2754-2787 (2008).

4 Tschierske, C. Micro-segregation molecular shape and molecular topology-partners for the design of liquid crystalline materials with complex mesophase morphologies. J. Mater. Chem 11, 2647-2671 (2001)

5 Bosman, A. W., Janssen, H. M. \& Meijer, E. W. About dendrimers: structure, physical properties, and applications. Chem. Rev. 99, 1665-1688 (1999).

6 Hawker, C. J., Malmström, E. E., Frank, C. W. \& Kampf, J. P. Exact linear analogs of dendritic polyether macromolecules: design, synthesis, and unique properties. J. Am Chem. Soc. 119, 9903-9904 (1997).

7 Grayson, S. M. \& Fréchet, J. M. J. Convergent dendrons and dendrimers: from synthesis to applications. Chem. Rev. 101, 3819-3868 (2001).

8 Rosen, B. M., Wilson, C. J., Wilson, D. A., Peterca, M., Imam, M. R. \& Percec, V. Dendron-mediated self-assembly, disassembly, and self-organization of complex systems. Chem. Rev. 109, 6275-6540 (2009).

9 Cho, B. -K., Jain, A., Nieberle, J., Mahajan, S. \& Wiesner, U. Synthesis and self assembly of amphiphilic dendrimers based on aliphatic polyether-type dendritic cores. Macromolecules 37, 4227-4234 (2004).

10 Chung, Y. -W., Lee, B. -I., Kim, H. -Y., Wiesner, U. \& Cho, B. -K. Influence of crystalline peripheral chain length on the solid-state assemblies of amphiphilic dendrons. J. Polym. Sci. A Polym. Chem 45, 4988-4994 (2007).

11 Chung, Y.-W., Lee, B. -I. \& Cho, B. -K. Thermal and solid state assembly behavior of amphiphilic aliphatic polyether dendrons with octadecyl peripheries. Macromol. Res. 16, 113-119 (2008).

12 Iyer, J. \& Hammond, P. T. Langmuir behavior and ultrathin films of new linear-dendritic diblock copolymers. Langmuir 15, 1299-1306 (1999).

13 Román, C., Fischer, H. R. \& Meijer, E. W. Microphase separation of diblock copolymers consisting of polystyrene and acid-functionalized poly(propylene imine) dendrimers. Macromolecules 32, 5525-5531 (1999).

14 Johnson, M. A., lyer, J. \& Hammond, P. T. Microphase segregation of PEO-PAMAM linear-dendritic diblock copolymers. Macromolecules 37, 2490-2501 (2004)

15 Leduc, M. R., Hawker, C. J., Dao, J. \& Fréchet, J. M. J. Dendritic initiator for 'Living' radical polymerizations: a versatile approach to the synthesis of dendritic-linear block copolymers. J. Am. Chem. Soc. 118, 11111-11118 (1996).

16 Gitsov, I. \& Fréchet, J. M. J. Novel nanoscopic architectures. Linear-globular ABA copolymers with polyether dendrimers as A blocks and polystyrene as B block. Macromolecules 27, 7309-7315 (1994).

17 Zhao, Y. -L., Jiang, J., Liu, H. -W., Chen, C. -F. \& Xi, F. Atom transfer radical copolymerization of $\mathrm{N}$-phenylmaleimide and styrene initiated with dendritic polyarylether 2-bromoisobutyrate. J. Polym. Sci. A Polym. Chem 39, 3960-3966 (2001).

18 van Hest, J. C. M., Delnoye, D. A. P., Baars, M. W. P. L., Van Genderen, M. H. P. \& Meijer, E. W. Polystyrene-dendrimer amphiphilic block copolymers with a generationdependent aggregation. Science 268, 1592-1595 (1995).

19 lyer, J., Fleming, K. \& Hammond, P. T. Synthesis and solution properties of new lineardendritic diblock copolymers. Macromolecules 31, 8757-8765 (1998)

20 Tian, L. \& Hammond, P. T. Comb-dendritic block copolymers as tree-shaped macromolecular amphiphiles for nanoparticle self-assembly. Chem. Mater. 18, 3976-3984 (2006).

21 Hillmyer, M. A., Bates, F. S., Almdal, K., Mortensen, K., Ryan, A. J. \& Fairclough, J. P. A. Complex phase behavior in solvent-free nonionic surfactants. Science 271, 976-978 (1996)

22 Cho, B. -K., Jain, A., Gruner, S. M. \& Wiesner, U. Mesophase structure-mechanical and ionic transport correlations in extended amphiphilic dendrons. Science 305, 15981601 (2004).

23 Grason, G. M. \& Kamien, R. D. Interfaces in diblcoks: a study of miktoarm sta copolymers. Macromolecules 37, 7371-7380 (2004)

24 Balagurusamy, V. S. K., Ungar, G., Percec, V. \& Johansson, G. Rational design of the first spherical supramolecular dendrimers self-organized in a novel thermotropic cubic liquid-crystalline phase and the determination of their shape by X-ray analysis. J. Am. Chem. Soc. 119, 1539-1555 (1997)

25 Percec, V., Ahn, C. -H., Ungar, G., Yeardley, D. J. P., Möller, M. \& Sheiko, S. S. Controlling polymer shape through the self-assembly of dendritic side-groups. Nature 391, 161-164 (1998).

26 Hamley, I. W. The Physics of Block Copolymers (Oxford Univ. Press, New York, 1998).
27 Chung, Y. -W., Lee, J. -K., Zin, W. -C. \& Cho, B. -K. Self-assembling behavior of amphiphilic dendron-coils in the bulk crystalline and liquid crystalline states. J. Am. Chem. Soc. 130, 7139-7147 (2008).

28 Lee, E., Lee, B. -I., Kim, S. -H., Lee, J. -K., Zin, W. -C. \& Cho, B. -K. Complex therma and bulk assembling properties of dendritic-linear-dendritic triblock copolymers depending on the length of the middle block. Macromolecules 42, 4134-4140 (2009).

29 Sun, L., Liu, Y., Zhu, L., Hsiao, B. \& Avila-Orta, C. A. Self-assembly and crystallization behevior of a double-crystalline polyethylene-block-poly(ethylene oxide) diblock copolymer. Polymer 45, 8181-8193 (2004).

30 Dormidontova, E. E. \& Lodge, T. P. The order-disorder transition and the disordered micelle regime in sphere-forming block copolymer melts. Macromolecules $\mathbf{3 4}$, 9143-9155 (2001).

31 Tarascon, J. -M. \& Armand, M. Issues and challenges facing rechargeable lithium batteries. Nature 414, 359-367 (2001).

32 Yoshio, M., Kagata, T., Hoshino, K., Mukai, T., Ohno, H. \& Kato., T. One-dimensiona ion-conductive polymer film: alignment and fixation of ionic channels formed by selforganization of polymerizable columnar liquid crystals. J. Am. Chem. Soc. 128, 5570-5577 (2006)

33 Yazaki, S., Kamikawa, Y., Yoshio, M., Hamasaki, A., Mukai, T., Ohno, H. \& Kato, T. Ionic liquid crystals: self-assembly of imidazolium salts containing an L-glutamic acid moiety. Chem. Lett. 37, 538-539 (2008).

34 Shimura, H., Yoshio, M., Hoshino, K., Mukai, T., Ohno, H. \& Kato, T. Noncovalent approach to one-dimensional ion conductors: enhancement of ionic conductivities in nanostructured columnar liquid crystals. J. Am. Chem. Soc. 130, 1759-1765 (2008).

35 Yoshio, M., Mukai, T., Ohno, H. \& Kato, T. One-dimensional ion transport in selforganized columnar ionic liquids. J. Am. Chem. Soc. 126, 994-995 (2004).

36 Ichikawa, T., Yoshio, M., Hamasaki, A., Mukai, T., Ohno, H. \& Kato, T. Self-organization of room-temperature ionic liquids exhibiting liquid-crystalline bicontinuous cubic phases: formation of nano-ion channel networks. J. Am. Chem. Soc. 129, 10662-10663 (2007)

37 Kerr, R. L., Miller, S. A., Shoemaker, R. K., Elliott, B. J. \& Gin, D. L. New type of Li ion conductor with $3 \mathrm{D}$ interconnected nanopores via polymerization of a liquid organic electrolyte-filled lyotropic liquid-crystal assembly. J. Am. Chem. Soc. 131, 15972-15973 (2009).

38 Song, J. Y., Wang, Y. Y. \& Wan., C. C. Review of gel-type polymer electrolytes for lithium-ion batteries. J. Power Sources 77, 183-197 (1999).

39 Choi, J. -W. \& Cho, B. -K. Mesomorphic and conducting properties of dendritic-linear copolymers via ion-doped additives. J. Polym. Sci. A Polym. Chem. 49, 2468-2473 (2011)

40 Epps, T. H., Bailey, T. S., Waletzko, R. \& Bates, F. S. Phase behavior and block sequence effects in lithium perchlorate-doped poly(isoprene- $b$-styrene- $b$-ethylene oxide) and poly(styrene- $b$-isoprene- $b$-ethylene oxide) triblock copolymers. Macromolecules 36, 2873-2881 (2003)

41 Kim, H. -Y., Song, J., Kim, S. -H., Lee, E., Lee, J. -K., Zin, W. -C. \& Cho, B. -K Hydrophilic matrix-assisted ionic transportation in the columnar assembly of amphiphilic dendron-coils. Chem. Eur. J. 15, 8683-8686 (2009).

42 Kim, H. -Y., Lee, B. -I. \& Cho, B. -K. Large-scale synthesis of water soluble non-ionic dendrons by stepwise extraction purifications. Macromol. Rapid Commun. 29, 1758-1763 (2008)

43 Percec, V., Imam, M. R., Bera, T. K., Balagurusamy, V. S. K., Peterca, M. \& Heiney, P. A. Self-assembly of semifluorinated Janus-dendritic benzamides into bilayered pyramidal columns. Angew. Chem. Int. Ed. 44, 4739-4745 (2005).

44 Bury, I., Heinrich, B., Bourgogne, C., Guillon, D. \& Donnio, B. Supramolecular selforganization of 'Janus-like' diblock codendrimers: synthesis, thermal behavior, and phase structure modeling. Chem. Eur. J. 12, 8396-8413 (2006).

45 Saez, I. M. \& Goodby, J. W. 'Janus' supramolecular liquid crystals-giant molecules with hemispherical architectures. Chem. Eur. J. 9, 4869-4877 (2003).

46 Nierengarten, J. -F., Eckert, J. -F., Rio, Y., Carreon, M. P., Gallani, J. -L. \& Guillon, D. Amphiphilic diblock dendrimers: synthesis and incorporation in Langmuir and Langmuir-Blodgett films. J. Am. Chem. Soc. 123, 9743-9748 (2001).

47 Ropponen, J., Nummelin, S. \& Rissanen, K. Bisfunctionalized janus molecules. Org. Lett. 6, 2495-2497 (2004).

48 Luman, N. R. \& Grinstaff. Synthesis and aqueous aggregation properties of amphiphilic surface-block dendrimers. Org. Lett. 7, 4863-4866 (2005).

49 Yuan, F., Wang, W., Yang, M., Zhang, X., Li, J., Li, H., He, B., Minch, B., Lieser, G. \& Wegner, G. Layered structure and order-to-disorder transition in a block codendrime caused by intermolecular hydrogen bonds. Macromolecules 39, 3982-3985 (2006).

50 Hernández-Ainsa, S., Marcos, M., Barberá, J. \& Serrano, J. L. Philic and phobic segregation in liquid-crystal ionic dendrimers: an enthalpy-entropy competition. Angew. Chem. Int. Ed. 49, 1990-1994 (2010).

51 Choi, J. -W., Ryu, M. -H., Lee, E. \& Cho, B. -K. Ion-induced bicontinuous cubic and columnar liquid-crystalline assemblies of discotic block codendrimers. Chem. Eur. J. 16, 9006-9009 (2010).

52 Rios, G. E. \& Pickett, G. T. Dendrimer-dendrimer copolymer melts. Macromolecules 36, 2967-2976 (2003)

53 Percec, V., Heck, J. A., Tomazos, D. \& Ungar, G. The influence of the complexation of sodium and lithium triflate on the self-assembly of tubular-supramolecular architectures displaying a columnar mesophase based on taper-shaped monoesters of oligoethylene oxide with 3,4,5-tris[p-(n-dodecan-1-yloxy)benzyloxy]benzoic acid and of their polymethacrylates. J. Chem. Soc. Perkin Trans. 2, 2381-2388 (1993).

54 Kölbel, M., Beyersdorff, T., Tschierske, C., Diele, S. \& Kain, J. Thermotropic and lyotropic liquid crystalline phases of rigid aromatic amphiphiles. Chem. Eur. J. 6 3821-3837 (2000) 
55 Choi, J. -W. \& Cho, B. -K. Degree of chain branching-dependent assemblies and conducting behavior in ionic liquid crystalline Janus dendrimers. Soft Matter 7, 4045-4049 (2011).

56 Rostovtsev, V. V., Green, L. G., Fokin, V. V. \& Sharpless, K. B. A stepwise Huisgen cycloaddition process: Copper(I)-catalyzed regioselective 'ligation' of azides and terminal alkynes. Angew. Chem. Int. Ed. 41, 2596-2599 (2002).

57 Gallardo, H., Ely, F., Bortoluzzi, A. J. \& Conte, G. Applying click chemistry to zsynthesis of chiral [1,2,3]-triazole liquid crystals. Liquid Crystal 32, 667-671 (2005).

58 Xia, Y., Verduzco, R., Grubbs, R. H. \& Kornfield, J. A. Well-defined liquid crystal gels from telechelic polymers. J. Am. Chem. Soc. 130, 1735-1740 (2008).

59 Srividhya, D., Manjunathan, S., Thirumaran, S., Saravanan, C. \& Senthil, S. Synthesis and characterization of $[1,2,3]$-triazole containing liquid crystals through click reaction. J. Mol. Struct. 927, 7-13 (2009).

60 Ryu, M. -H., Choi, J. -W. \& Cho, B. -K. Design, synthesis, and self-assembly behavior of $C_{3}$-symmetry discotic molecules via click chemistry. J. Mater. Chem. 20, 1806-1810 (2010).

61 Kroon, J. M., Koehorst, R. B. M., van Dijk, M., Sanders, G. M. \& Sudhölter, J. R. Selfassembling properties of non-ionic tetraphenylporphyrins and discotic phthalocyanines carrying oligo(ethylene oxide) alkyl or alkoxy units. J. Mater. Chem. 7, 615-624 (1997).

62 Bock, H., Babeau, A., Seguy, I., Jolinat, P. \& Destruel, P. Electron-deficient columnar plastic crystals. ChemPhysChem 3, 532-535 (2002).

63 Kraft, A., Reichert, A. \& Kleppinger, R. Supramolecular liquid crystals with columnar mesophases through self-assembly of carboxylic acids around a tribasic core. Chem. Commun. 1015-1016 (2000).

64 Kleppinger, R., Lillya, C. P. \& Yang, C. Self-assembling discotic mesogens. Angew. Chem. Int. Ed. 34, 1637-1638 (1995).

65 Suárez, M., Lehn, J. -M., Zimmerman, S. C. Skoulios, A \& Heinrich, B. Supramolecular liquid crystals. Self-assembly of a trimeric supramolecular disk and its selforganization into a columnar discotic mesophase. J. Am. Chem. Soc. 120, 95269532 (1998).

$66 \mathrm{Li}$, Y. \& Flood, A. H. Pure C-H hydrogen bonding to chloride ions: a preorganized and rigid macrocyclic receptor. Angew. Chem. Int. Ed. 47, 2649-2652 (2008).

67 Ryu, M. -H., Choi, J. -W., Kim, H. -J., Park, N. \& Cho, B. -K. Complementary hydrogen bondings between a clicked $C_{3}$-symmetry triazole derivative and carboxylic acids for columnar liquid crystalline assemblies. Angew. Chem. Int. Ed. 50, 5737-5740 (2011). 